\title{
Inverse Diffraction Theory and Computation of Minimum Source Regions of Far Fields
}

\author{
Edwin A. Marengo \\ Department of Electrical and Computer Engineering, Northeastern University, Boston, MA 02115, USA \\ Correspondence should be addressed to Edwin A. Marengo; emarengo@ece.neu.edu
}

Received 29 August 2013; Revised 29 October 2013; Accepted 5 November 2013; Published 12 January 2014

Academic Editor: Davide La Torre

Copyright (C) 2014 Edwin A. Marengo. This is an open access article distributed under the Creative Commons Attribution License, which permits unrestricted use, distribution, and reproduction in any medium, provided the original work is properly cited.

\begin{abstract}
A methodology based on the multipole expansion is developed to estimate the minimum source region of a given far field. The support of any source that produces the given far field must contain this minimum source region. The results are derived in the framework of the scalar Helmholtz equation in two-dimensional free space, which is relevant to transverse magnetic electromagnetic waves. The proposed approach consists of two steps. First we address, via an exterior inverse diffraction framework, the estimation of the minimum convex source region, which is the convex hull of the minimum source region. Next we compute, via a complementary interior inverse diffraction approach, nonconvex bounds for the minimum source region. This allows, in theory, the estimation of the minimum source region which can be nonconvex. The derived approach is illustrated with analytical and numerical examples relevant to inverse source and scattering problems.
\end{abstract}

\section{Introduction}

We consider in the framework of the scalar Helmholtz equation the inverse problem of estimating the minimum source region of a given far field. The support of any source that produces the given far field must contain this minimum source region. This region is also sufficiently big so that there exists a source supported in it that radiates the given far field. Thus this region is optimal in the sense that it is the smallest support of any source that can produce the given far field. Previous works tackling this fundamental inverse problem have emphasized the computation of the convex hull of the minimum source region, which has been termed the convex scattering support [1] or the minimum convex source region [2] of the given far field. The purpose of the present paper is to further extend the scope of the available theory and algorithms to compute the actual minimum source region including nonconvex minimum source regions that cannot be estimated with the existing methods. We also derive new closed form formulas for the minimum source region that are based on the multipole expansion [3] and that complement the counterpart plane-wave-theorybased, closed form method of a previous paper [2]. The derived formulas facilitate analytical demonstration of the computation of the minimum source region for far fields that are known in closed form, which is of interest in antenna synthesis and as a theoretical tool to handle certain inverse scattering problems analytically. We also address the respective numerical implementation and illustrate it with examples. The results are quite encouraging, rendering good bounds of source support for the particular sources considered. On the other hand, we do not specify in the following the class of sources for which the methods of this paper (the nonconvex bounds, in particular) are useful in practice. This challenging question is related to theoretical issues raised in [4] which go beyond the scope of our presentation and is left for the future.

Next we outline the key background and ideas and sketch the plan of this paper. In this introduction, we outline the problem statement implicitly assuming the physical threedimensional (3D) space. However, to facilitate the subsequent exposition as well as the tractability of illustrative examples, in the following sections of the paper we restrict attention to the simpler two-dimensional (2D) formulation.

It has been shown in a number of investigations that, given the far field associated with an unknown radiating source, it is possible to uniquely define and compute the so-called convex scattering support [1] or minimum convex 


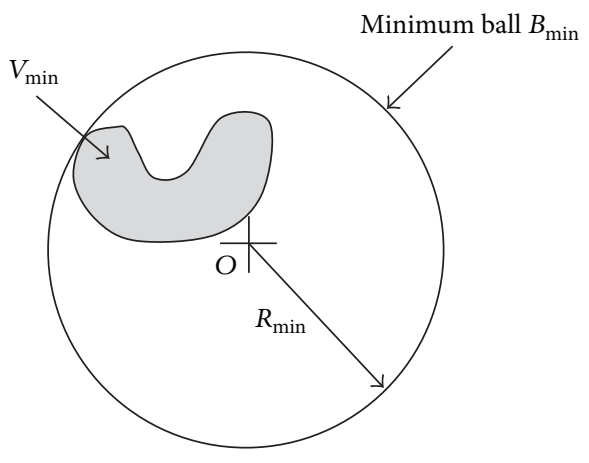

FIGURE 1: Minimum source region $V_{\min }$ and the minimum ball $B_{\text {min }}(O)$ for a given origin $O$.

source region [2] associated with the given far field. The theoretical principles can be traced back to the treatise of Müller [5, Theorems 26, 27, 29] who used the spherical wave or multipole expansion of the radiated field to show that, for a fixed origin of coordinates, say $O$, there is a minimum spherical source region of radius $R_{\min }, B_{\min }(O)=\{\mathbf{r} \epsilon$ $\left.\mathbb{R}^{3}: r \equiv|\mathbf{r}| \leq R_{\text {min }}(O)\right\}$, such that, in order for a source to be able to produce the given far field radiation pattern, it must lie in the interior of a spherical volume centered about the same origin and having a radius that is at least as large as $R_{\min }$. Furthermore, the radiated field outside $B_{\text {min }}$ is an analytic function that can be analytically continued up to the boundary of a minimum source region $V_{\text {min }}$ located inside $B_{\text {min }}$; see [6, page 143]. Figure 1 illustrates the minimum source region and the respective minimum spherical source region corresponding to a given origin. From the point of view of propagation or diffraction, then the far field can be inverse-diffracted up to the boundary of $V_{\min } \subseteq B_{\min }$. In addition, clearly if one considers another origin $O^{\prime}$ then one can also find another minimum spherical volume $B_{\min }\left(O^{\prime}\right)$ centered about this origin, and so on. Then if one considers a set of, say, $n$ origins and $n$ associated minimum spherical volumes, the intersection of all such source regions, say $\mathscr{B}_{\text {min }}$, establishes a sharper bound on the minimum source region $V_{\text {min }} \subseteq \mathscr{B}_{\text {min }}$ which lies in the interior of such intersection. By means of this procedure, which has been elaborated in rigor in $[1,7]$, it is theoretically possible to uniquely define and compute the convex scattering support or minimum convex source region, say $B_{\text {min,conv }} \supseteq V_{\text {min }}$, which is a subset of the convex hull of any source producing the given far field. This minimum convex source region is the smallest convex support of any source producing that field and represents important source localization information that can be inverted uniquely from the far field data despite the general nonuniqueness (without further priors) of the full inverse source problem of reconstructing the actual source that generated the given far field; see, for example, $[8,9]$ for discussions of the inverse source problem and the relevant nonradiating sources corresponding to the null space of the forward source-to-field mapping. In this connection, it is worth commenting that for zero far fields the minimum source region does not exist (empty set). Any nonradiating source can produce that trivial far field, and of course no source at all also produces that same far field. However, for nontrivial far fields associated with fields obeying the radiation condition the minimum source region is a nonempty set since, as is well known, the only solution to the homogeneous Helmholtz equation obeying the radiation condition is the zero field. Consequently, here and henceforth we implicitly assume nontrivial far fields, for which one can always define corresponding minimum source regions.

The theory behind the convex scattering support or minimum convex source region has been detailed in the key papers $[1,4,7,10,11]$. Kusiak and Sylvester [1] used the circular Paley-Wiener theorem to demonstrate the existence and computation of the convex scattering support. The original focus was, as in [2], on wave radiation in free space. Potthast et al. [10] proposed the "range test" to compute in practice the convex scattering support. Kusiak and Sylvester [7] demonstrated that the convex scattering support can be defined via a Picard test. This is an important generalization which allows the definition of the convex support (of the object function) for a broad class of inverse problems, including inverse source and scattering problems in nonhomogeneous media, and problems with limited data. Sylvester [4] demonstrated the existence of a unique union of well-supported convex sets (UWSC sets) corresponding to the given far field. A source having that support exists that radiates the given far field. Also, this unique UWSC sets is a subset of the UWSC support of any source that produces the same far field. Haddar et al. [11] addressed the related problem of computing the minimum convex support of a scatterer from back-scattering data. Most of these studies were clearly motivated by the relevance of the convex scattering support in inverse scattering problems, with linkages to the sampling and probe methods [12].

The computation of the minimum convex source region has also been addressed by means of a simpler methodology based on the plane wave expansion, rendering an alternative approach to estimate the minimum convex source region of a given far field ([2]; see also [6, pages 143-146]). Unlike the multipole expansion or circular Paley-Wiener theorem approaches, or other range or Picard tests proposed to date, the plane wave expansion method in [2] does not depend on the position of the origin of the coordinate system. On the other hand, this method is useful only if the far field is known in closed form. For real data and computations, one needs to adopt alternative approaches such as those detailed in $[1,7]$ and other related studies $[10,11]$. Nevertheless, from both theoretical and practical points of view, there is a lot of interest in the analytical approach in [2] since it allows handling of problems where the far field is known in closed form, which cannot be studied in rigor with computational approaches which employ discrete data.

The method in [2] applies to the minimum convex source region. For geometrical reasons this plane wave expansion approach cannot be used to estimate nonconvex minimum source regions. In this paper we use the multipole expansion to obtain the respective closed form formula to estimate the full minimum source region including nonconvex minimum source regions that cannot be derived with the existing 
methods. To achieve this goal, first we derive, in $2 \mathrm{D}$ space, the respective multipole theory counterpart of the key formula (6) in [2], for estimation of the convex support. Later we derive the respective generalization to tackle the pending nonconvex support, which completes the computation of the minimum source region. In addition to studying the analytical computation of minimum source regions, we also develop and illustrate with examples the corresponding numerical version of the approach.

We wish to point out that part of our motivation to generalize the estimation of the minimum convex source region to the actual minimum source region is provided by the generality of the Picard test as shown in [7] and the related paper [10]. We show that, in theory, just as we can estimate from the far field the respective minimum convex source region via a Picard test based on an exterior inverse problem from far fields to sources in the near field region, we can also formulate the estimation of the possibly nonconvex minimum source region via a similar Picard test based on an interior inverse problem involving near fields. In the exterior problem one goes from the field data in a large spherical surface to a source contained inside a spherical volume of smaller radius. In the interior problem one goes from the near field data in a small spherical surface to a source supported outside a spherical volume of larger radius. In problems involving real data, the near field can be estimated from the far field only up to a finite-dimensional approximation due to the ill-posedness of the near-to-far field map. However, it may be possible, even in that realistic computational framework, to make an estimate of the possibly nonconvex minimum source region from the far field data. The evanescent near field components decay a short distance (a wavelength or two) away from the source, so that the inverse diffraction of the far field back to the near zone remains reliable up to a short distance away from the source, and consequently the inference of some nonconvex support information is possible as we show in the paper. For far fields that are known in closed form, the near field can be computed uniquely up to the minimum convex source region and the minimum source region can be estimated subsequently via the proposed approach.

We wish to further comment on the fundamental and practical relevance of minimum source regions. The last decade has seen the development of many methods for imaging and shape reconstruction of scatterers from scattering data. The efforts on minimum source regions are an important part of the associated state-of-the-art for a number of reasons. First, the definition of the minimum source region of a far field is rigorous. It sheds light on the information about the support of a source or scatterer that is contained in far field data. Even though the inverse source problem exhibits general nonuniqueness, the minimum source region is a piece of information about the sought-after source that can be deduced uniquely from the far field. In relation to alternative imaging methods, this approach has the particular appeal that it applies in theory to the extraction of information about the support of a scatterer using a single probing field. In numerical scattering experiments, this method is quite robust $[7,10,11]$, even though it defines only a bound for the true scatterer support. Finally, thanks to the generalization in [7], perhaps the main appeal of this theory is its universality, suggesting its implementation as both a theoretical tool and an algorithm in a broad class of inverse problems.

The rest of the paper is organized as follows. Section 2 presents, in the framework of the Helmholtz equation in 2D space, the key theoretical results for estimating bounds for the minimum convex source region as well as the possibly nonconvex minimum source region. First we address the estimation of convex bounds, in theory up to the minimum convex source region. This is done via an exterior inverse diffraction approach. Next we refine the estimate by allowing nonconvex bounds, in theory up to the actual minimum source region which can be nonconvex. This is done by means of a complementary, interior inverse diffraction approach. In Section 3 we consider examples motivated by inverse scattering that can be handled analytically. Of particular interest is the support estimation of cylindrical objects, within exact scattering theory, from the scattering amplitude of a single scattering experiment (for a single probing field). In Section 4 we discuss the practical computational implementation aspects of the inverse support methods derived in the paper and present the numerical counterpart of the analytical results obtained in Section 3. The obtained computational results show the practical robustness of minimum source region computations in inverse scattering. In addition, the provided examples nicely illustrate the UWSC sets theorized in earlier work [4]. Section 5 provides concluding remarks and suggestions for future research.

\section{Theory}

2.1. Forward Problem. We consider electromagnetic radiation and scattering problems involving transverse magnetic $z\left(\mathrm{TM}^{z}\right)$ polarization for which radiation is governed by the Helmholtz equation in 2D free space,

$$
\left(\nabla^{2}+k^{2}\right) E_{z}(\mathbf{r})=-i \omega \mu I_{z}(\mathbf{r}),
$$

where $E_{z}$ is the $z$ component of the electric field, $I_{z}$ is the $z$ component of the current distribution that produces the field, $\omega$ is the angular oscillation frequency, $\mu$ is the free space permeability, and $k$ is the wavenumber, defined by

$$
k=\omega \sqrt{\mu \epsilon},
$$

where $\epsilon$ is the free space permittivity. In Cartesian coordinates $\mathbf{r}=(x, y)$ while in cylindrical coordinates $\mathbf{r}=(\rho, \phi)$ where $\rho \equiv|\mathbf{r}|$ and $\phi$ is the azimuthal angle. This context is relevant, for example, in wave modeling of long cylindrical objects (relative to the wavelength $\lambda=2 \pi / k$ ) that essentially depend only on the transverse coordinates. The source $I_{z}$ can represent the transverse dependence of an active source (antenna) or an induced source such as a scatterer excited by incident fields.

The radiated field obeying the radiation condition at infinity is well known to be given by [13, page 598]

$$
E_{z}(\mathbf{r})=-\frac{\omega \mu}{4} \int d \mathbf{r}^{\prime} H_{0}^{(1)}\left(k\left|\mathbf{r}-\mathbf{r}^{\prime}\right|\right) I_{z}\left(\mathbf{r}^{\prime}\right),
$$


where $H_{0}^{(1)}$ is the Hankel function of the first kind and order 0 . From the addition theorem for Hankel functions we have

$$
H_{0}^{(1)}\left(k\left|\mathbf{r}-\mathbf{r}^{\prime}\right|\right)=\sum_{m=-\infty}^{\infty} J_{m}\left(k \rho_{<}\right) H_{m}^{(1)}\left(k \rho_{>}\right) e^{-i m\left(\phi-\phi^{\prime}\right)}
$$

where $\rho_{<}=\min \left(\rho, \rho^{\prime}\right)$ and $\rho_{>}=\max \left(\rho, \rho^{\prime}\right)$ where $\rho$ is the distance from $\mathbf{r}$ to the origin of coordinates and $\rho^{\prime}$ is the distance from $\mathbf{r}^{\prime}$ to the origin.

For a source whose support $V$ is confined inside a disk or 2 -ball of radius $R$ centered about the origin $O$ (see Figure 2 ) we have from (3) and (4)

$$
E_{z}(\mathbf{r}=(\rho, \phi))=-\frac{\omega \mu}{4} \sum_{m=-\infty}^{\infty} a_{m} H_{m}^{(1)}(k \rho) e^{-i m \phi} \quad \rho>R,
$$

where the multipole moments

$$
a_{m}=\int_{0}^{R} d \rho^{\prime} \rho^{\prime} \int_{0}^{2 \pi} d \phi^{\prime} J_{m}\left(k \rho^{\prime}\right) e^{i m \phi^{\prime}} I_{z}\left(\rho^{\prime}, \phi^{\prime}\right) .
$$

Using the large argument approximation for the Hankel function

$$
H_{m}^{(1)}(k \rho) \sim \sqrt{\frac{2}{\pi k \rho}} e^{i[k \rho-m(\pi / 2)-\pi / 4]}
$$

we get from (14) the far zone behavior

$$
E_{z}(\rho, \phi) \sim-\frac{\omega \mu}{4} \sqrt{\frac{2}{\pi k \rho}} e^{i(k \rho-\pi / 4)} f(\phi),
$$

where the far field radiation pattern

$$
f(\phi)=\sum_{m=-\infty}^{\infty} i^{-m} a_{m} e^{-i m \phi}
$$

so that in view of the orthogonality of the complex exponentials for integer $m$

$$
a_{m}=\frac{i^{m}}{2 \pi} \int_{0}^{2 \pi} d \phi e^{i m \phi} f(\phi) .
$$

It can be shown from (8) and (9), Faraday's law, and Poynting's theorem that the average radiated power $P$ is given by

$$
P=\frac{\omega \mu}{8} \sum_{m=-\infty}^{\infty}\left|a_{m}\right|^{2}<\infty
$$

which must be finite, implying that the multipole moments are square-summable.

In addition, for nontrivial far fields, there exists a source inside the minimum source region $V_{\text {min }} \subseteq V$ (see Figure 2) that generates the given far field, corresponding to the given multipole moments $a_{m}$. By applying the above formulation to this source we obtain

$$
E_{z}(\mathbf{r}=(\rho, \phi))=-\frac{\omega \mu}{4} \sum_{m=-\infty}^{\infty} a_{m} H_{m}^{(1)}(k \rho) e^{-i m \phi} \quad \rho>R_{\min },
$$

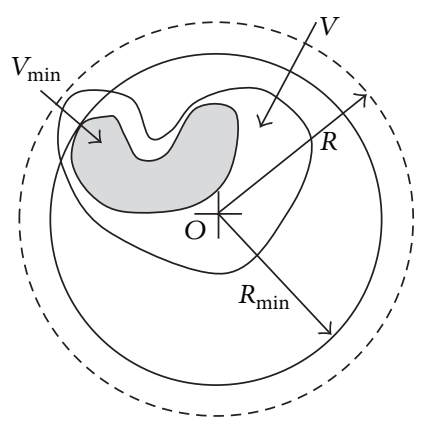

Figure 2: Relations between $V_{\min }$ and $V$ and $R_{\min }$ and $R$ for a given origin $O$.

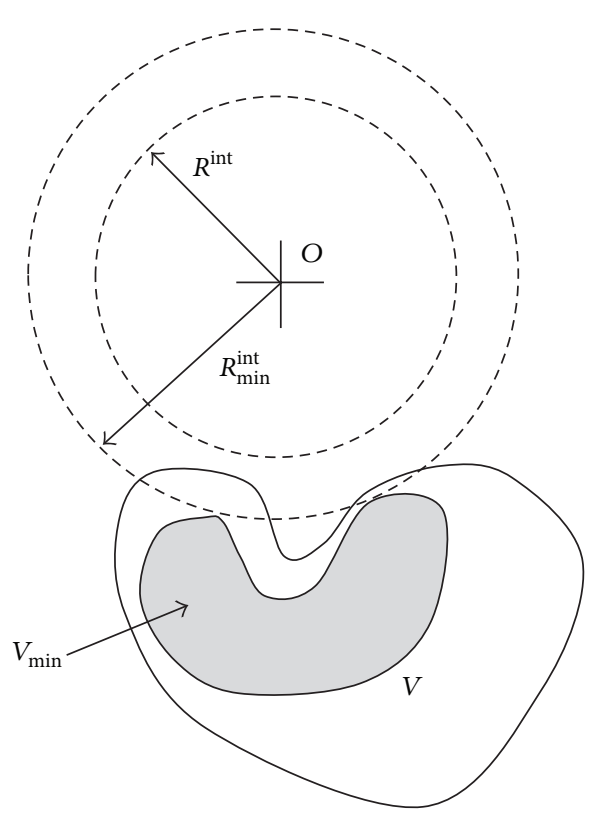

FIgURE 3: Relations between $V_{\min }$ and $V$ and $R_{\min }^{\text {int }}$ and $R^{\text {int }}$ for a given origin $O$.

where $R_{\min }$ is the radius of the smallest ball that is centered about the same origin and contains the minimum source region $V_{\min } \subseteq V$ of the given far field (see Figure 2).

On the other hand, if the origin of coordinates $O$ is such that $V$ is contained outside a circle of radius $R^{\text {int }}$ centered at the origin (see Figure 3), then the field in the interior of this circle of radius $R^{\text {int }}$ is given from (3) and (4) by

$$
E_{z}(\mathbf{r}=(\rho, \phi))=-\frac{\omega \mu}{4} \sum_{m=-\infty}^{\infty} g_{m} J_{m}(k \rho) e^{-i m \phi} \quad \rho<R^{\mathrm{int}},
$$

where

$$
g_{m}=\int_{R^{\mathrm{int}}}^{\infty} d \rho^{\prime} \rho^{\prime} \int_{0}^{2 \pi} d \phi^{\prime} H_{m}^{(1)}\left(k \rho^{\prime}\right) e^{i m \phi^{\prime}} I_{z}\left(\rho^{\prime}, \phi^{\prime}\right) .
$$


Furthermore, there is a source inside $V_{\text {min }} \subseteq V$ that generates the same field outside $V$ so that

$$
E_{z}(\mathbf{r}=(\rho, \phi))=-\frac{\omega \mu}{4} \sum_{m=-\infty}^{\infty} g_{m} J_{m}(k \rho) e^{-i m \phi} \quad \rho<R_{\min }^{\mathrm{int}},
$$

where $R_{\mathrm{min}}^{\mathrm{int}}$ is the radius of the largest ball centered about the same origin that is disjoint to the minimum source region $V_{\min }$ and is tangential to it in a way that defines a concave boundary of $V_{\min }$. This is illustrated in Figure 3.

2.2. Minimum Convex Source Region. Let us derive next convex and nonconvex boundaries for the minimum source region of a given far field based on the results above. We tackle first the convex support information by means of a Picard test applied to an exterior inverse diffraction problem.

Equations (8) and (9) define the far field while the field for $\rho=a>R$ is defined by (5); that is,

$$
E_{z}(a, \phi)=-\frac{\omega \mu}{4} \sum_{m=-\infty}^{\infty} a_{m} H_{m}^{(1)}(k a) e^{-i m \phi} .
$$

Next, we inverse-diffract this boundary-value field to a more interior (smaller) circle of radius $\rho=b<a$, requiring that the resulting field for $\rho=b<a$ be square-integrable $\left(L^{2}\right)$ over the unit circle, which is the Picard condition of this linear inverse problem. The reconstructed field in the circle of radius $\rho=b<a$ that obeys the homogeneous form of (1) must be of the form

$$
E_{z}(b, \phi)=-\frac{\omega \mu}{4} \sum_{m=-\infty}^{\infty} a_{m} H_{m}^{(1)}(k b) e^{-i m \phi},
$$

but this is meaningful only if

$$
\sum_{m=-\infty}^{\infty}\left|a_{m}\right|^{2}\left|H_{m}^{(1)}(k b)\right|^{2}<\infty
$$

The smallest circle onto which the field can be inversediffracted via (16) and (17) defines the sought-after minimum circular region $B_{\min }$ associated with the given far field $f(\phi)$ or equivalently the multipole moments $a_{m}$ and is defined as $B_{\text {min }}=\left\{\mathbf{r} \in \mathbb{R}^{2}:|\mathbf{r}| \leq R_{\min }\right\}$ where

$$
R_{\min }=\inf \left\{b \in \mathbb{R}^{+}: \sum_{m=-\infty}^{\infty}\left|a_{m}\right|^{2}\left|H_{m}^{(1)}(k b)\right|^{2}<\infty\right\},
$$

where inf means infimum. The following result follows from (19) and the asymptotic behavior (see [5, page 77])

$$
H_{m}^{(1)}(z) \sim-\frac{i}{\pi} \Gamma(m)\left(\frac{2}{z}\right)^{m}, \quad m \longrightarrow \infty .
$$

Main Result 1. We have

$$
R_{\min }=\sup \left\{b \in \mathbb{R}^{+}: \lim _{m \rightarrow \infty} \Gamma(m)\left(\frac{2}{k b}\right)^{m}\left|a_{m}\right| \neq 0\right\} .
$$

Here sup means supremum. The proof is given in Appendix A.
Main Result 1(21) holds for any origin O. The intersection of the minimum spherical source regions $B_{\min }=\{\mathbf{r} \epsilon$ $\left.\mathbb{R}^{2}: \rho \leq R_{\min }\right\}$ for different origins defines a convex region where the minimum source region resides. It is theoretically possible for sufficiently many such origins to actually define the minimum convex source region $B_{\text {min,conv }}$ of the given far field. The method can be summarized as follows. (1) Consider a number $n$ of origins of coordinates, $O_{\alpha}, \alpha=1,2, \ldots, n$, within the region of interest. (2) For each origin, compute via the test (21) the associated minimum radius $R_{\min }\left(O_{\alpha}\right)$ and associated minimum circular region $B_{\min }\left(O_{\alpha}\right)=\left\{\mathbf{r} \in \mathbb{R}^{2}\right.$ : $\left.\left|\mathbf{r}-O_{\alpha}\right| \leq R_{\min }\left(O_{\alpha}\right)\right\}$. (3) The intersection of the regions $B_{\min }\left(O_{\alpha}\right), \alpha=1,2, \ldots, n$, defines a convex region $\mathscr{B}_{\min }$ bounding the minimum convex source region $B_{\text {min,conv }}$ in the sense that $B_{\text {min,conv }} \subseteq \mathscr{B}_{\text {min }}$. The estimate $\mathscr{B}_{\text {min }}$ becomes closer to $B_{\text {min,conv }}$ as one takes more sample origins and their corresponding minimum circular regions. Now, since (12) can be used for any of the test origins $O_{\alpha}$, it follows that we can uniquely associate, with the given far field, a valid radiated field that is well defined everywhere outside the respective convex region $\mathscr{B}_{\text {min }}$ that contains the minimum convex source region $B_{\text {min,conv }}$. We discuss next how to use this near field information to further refine our estimate of the minimum source region, in theory up to the possibly nonconvex minimum source region.

2.3. Inference of Nonconvex Support Information. In order to sharpen the estimate of the minimum source region, including nonconvex regions, we consider an interior inverse diffraction problem in the near field, associated with a complementary geometry where the field data are known in the interior of a given circle (interior data) and the field is inverse-diffracted into outer concentric circles. If closed form expressions for the far field or the multipole moments are available, then it is theoretically possible to estimate also the associated near field. If, on the other hand, the data are experimental or computational and thus subject to noise and perturbations, then the near field can be estimated only up to an essentially finite-dimensional representation, for example, the well known truncation $a_{m} \simeq 0$ for $m>k R$, where $R$ is the radius of the smallest circle that bounds the source support $V$. We assume next that the near field outside the convex region $\mathscr{B}_{\text {min }}$ can be estimated analytically and leave the practical question of estimating the near field computationally for other sections of this paper which address the computational implementation and examples.

Once we compute the convex region $\mathscr{B}_{\text {min }}$ that contains the minimum convex source region $B_{\text {min,conv }}$ as well as the radiated field that it produces in its exterior, it becomes possible to estimate another, possibly nonconvex region that is contained inside $\mathscr{B}_{\text {min }}$ and that contains the minimum source region. We assume that the field in the interior of a ball of radius $b$ centered at the origin can be estimated, for example, as part of the computations giving $\mathscr{B}_{\min }$. We use this interior field information to compute the radius $R_{\min }^{\text {int }}$ of the largest circle up to which said interior field can be inversediffracted. The geometry of this interior inverse diffraction can pierce or intersect concave portions of $B_{\text {min,conv }}$, allowing 


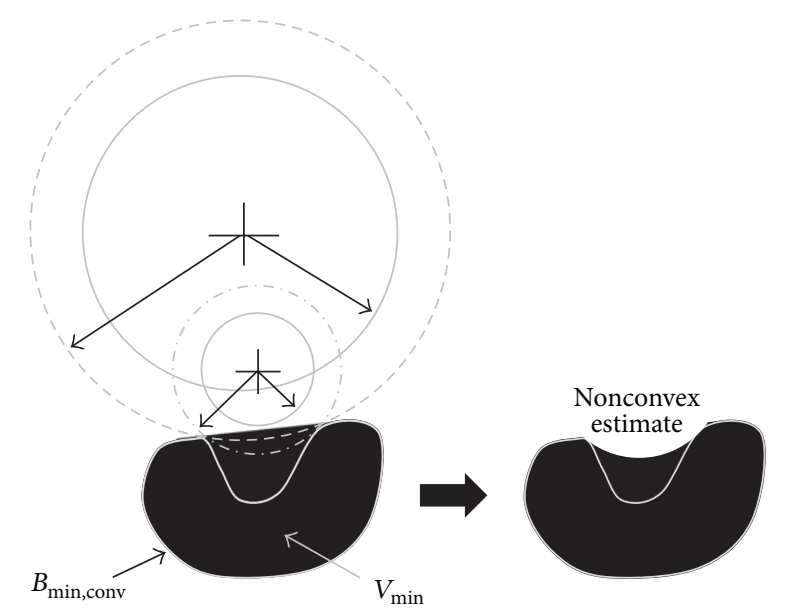

FIGURE 4: Successive application of the diffraction-based estimation of the critical sphere of radius $R_{\text {min }}^{\text {int }}$, based on field information available from previous iterations. This method allows the estimation of concave boundaries of the minimum source region. Thus it allows, in theory, the estimation of the minimum source region $V_{\min }$ which can be nonconvex.

the extraction of the pending nonconvex support information of $V_{\min }$ that is not contained in $B_{\text {min,conv }}$. The concept is illustrated in Figure 4.

In particular, consider for a given origin $O$, chosen outside the derived bounding region for the minimum convex source region, a ball centered at that origin and residing outside the bounding region for the minimum source region. It follows from (13) and (14) with the substitution of $V$ by the derived bounding region that in the interior of the circular region in question the field obeys an expansion of the form (13). The field at a circle of radius $\rho=b$ inside this region is then

$$
E_{z}(b, \phi)=-\frac{\omega \mu}{4} \sum_{m=-\infty}^{\infty} g_{m} J_{m}(k b) e^{-i m \phi} .
$$

Inverse diffraction to a more exterior circle of radius $\rho=a>$ $b$ is done via

$$
E_{z}(a, \phi)=-\frac{\omega \mu}{4} \sum_{m=-\infty}^{\infty} g_{m} J_{m}(k a) e^{-i m \phi}
$$

which is meaningful so long as

$$
\sum_{m=-\infty}^{\infty}\left|g_{m}\right|^{2}\left|J_{m}(k a)\right|^{2}<\infty
$$

The largest circle of radius $R_{\min }^{\text {int }}$ onto which the field can be inverse-diffracted this way defines a bounding region for the minimum source region associated with the given far field $f(\theta)$ or equivalently the multipole moments $a_{m}$. From (24)

$$
R_{\min }^{\mathrm{int}}=\sup \left\{a \in \mathbb{R}^{+}: \sum_{m=-\infty}^{\infty}\left|g_{m}\right|^{2}\left|J_{m}(k a)\right|^{2}<\infty\right\} .
$$

The minimum source region $V_{\text {min }} \subseteq \bar{B}_{\text {min }}^{\text {int }}$ where $\bar{B}_{\text {min }}^{\text {int }}=\{\mathbf{r} \epsilon$ $\left.\mathbb{R}^{2}: \mathbf{r} \notin B_{\min }^{\mathrm{int}}\right\}$.
The following result follows from (25) and the asymptotic property (see [5, page 71])

$$
J_{m}(z) \sim \frac{(z / 2)^{m}}{\Gamma(m+1)}, \quad m \longrightarrow \infty .
$$

Main Result 2. We have

$$
R_{\mathrm{min}}^{\mathrm{int}}=\inf \left\{a \in \mathbb{R}^{+}: \frac{\lim _{m \rightarrow \infty}(k a / 2)^{m}\left|g_{m}\right|}{\Gamma(m+1)} \neq 0\right\} .
$$

The proof uses the same general approach used to derive Main Result 1 in Appendix A and does not need to be (essentially) repeated.

The procedure to estimate the minimum source region can now be summarized as follows.

(1) Applying the exterior inverse diffraction approach of the previous subsection, compute the convex region $\mathscr{B}_{\text {min }}$ where $V_{\text {min }} \subseteq B_{\text {min,conv }} \subseteq \mathscr{B}_{\text {min }}$. For sufficiently many computational origins it is possible that the estimate $\mathscr{B}_{\text {min }}=$ $B_{\text {min,conv }}$.

(2) Consider next reference origins outside $\mathscr{B}_{\text {min }}$, say $O_{\alpha}, \alpha=1,2, \ldots, n$, and evaluate the field (via the exterior inverse diffraction approach) over circles centered about those origins and having radius as large as possible but not intersecting the convex region $\mathscr{B}_{\text {min }}$. Let us assume that such computational circles correspond to radii $b_{\alpha}$, with centers $O_{\alpha}$.

(3) Using the value of the field on such circles compute the respective minimum radius $R_{\min }^{\text {int }}(\alpha)$.

(4) Compute the union $U^{\mathrm{int}}$ of all the regions $B_{\min }^{\mathrm{int}}(\alpha)=$ $\left\{\mathbf{r} \in R^{2}:\left|\mathbf{r}-O_{\alpha}\right| \leq R_{\min }^{\text {int }}(\alpha)\right\}$. Compute the respective complement $\bar{U}^{\text {int }}=\left\{\mathbf{r} \in \mathbb{R}^{2}: \mathbf{r} \notin U^{\text {int }}\right\}$.

(5) Compute the intersection of $\bar{U}^{\text {int }}$ and $\mathscr{B}_{\text {min }}$, which defines the revised estimate $V_{\text {min,est }} \supseteq V_{\text {min }}$ for the minimum source region which can be nonconvex, in particular,

$$
V_{\text {min,est }}=\mathscr{B}_{\text {min }} \cap \bar{U}^{\text {int }} .
$$

(6) The information used in the previous steps is the field outside the convex region $\mathscr{B}_{\text {min }}$. However, as the estimate $V_{\text {min,est }}$ becomes closer to $V_{\text {min }}$ than $\mathscr{B}_{\text {min }}$ we need to repeat steps 2-4 for computational circles located outside the best current estimate $V_{\text {min,est }}$. Computation of the field outside the best current estimate relies on the interior inverse diffraction approach explained in this section. The counterpart of step 5 associated with the second and following iterations is $V_{\text {min,est }}$ (current) $=V_{\text {min,est }}$ (previous) $\cap \bar{U}^{\text {int }}$. By continuing this process (see Figure 4) it is theoretically possible to obtain $V_{\text {min,est }}=V_{\text {min }}$, as desired.

As an example of the implementation of this methodology, we discuss in Appendix B the computation of the minimum convex source region and minimum source region associated with the far field of two point sources and generalize to $N$ sources. In the following section we consider more examples, involving canonical scattering objects that can be addressed analytically. The complementary numerical counterpart of the same examples is presented in another section. 


\section{Analytical Scattering Examples}

3.1. Plane Wave Scattering by a Dielectric Circular Cylinder. To demonstrate the application of Main Result 1, we consider scattering by canonical objects for which analytical results are available. First we consider electromagnetic wave scattering by a lossless dielectric circular cylinder of radius $R$ centered about the origin. It is assumed that the cylinder's length is so much larger than the wavelength that it can be modelled as being infinitely long along the $z$-axis, which facilitates description of the corresponding wave radiation, propagation, and scattering via the 2D Helmholtz equation model. The relative permittivity and permeability of this cylinder are $\epsilon_{r}$ and $\mu_{r}$, respectively. The free space wavenumber $k$ is defined in (2). On the other hand, the dielectric wavenumber $k_{1}=k \sqrt{\mu_{r} \epsilon_{r}}$. It is well known that the field scattered in free space by this dielectric circular cylinder upon excitation by a $\mathrm{TM}^{z}$ plane wave of amplitude $E_{0}$ traveling in the $x$ direction can be written as ([14], page 666)

$$
E_{z}(\rho, \phi)=E_{0} \sum_{m=-\infty}^{\infty} a_{m} H_{m}^{(1)}(k \rho) e^{-i m \phi} \quad \rho>R
$$

which corresponds to the far field radiation pattern defined in (9) with

$$
a_{m}=i^{m} \frac{J_{m}^{\prime}(k R) J_{m}\left(k_{1} R\right)-\sqrt{\epsilon_{r} / \mu_{r}} J_{m}(k R) J_{m}^{\prime}\left(k_{1} R\right)}{\sqrt{\epsilon_{r} / \mu_{r}} J_{m}^{\prime}\left(k_{1} R\right) H_{m}^{(1)}(k R)-J_{m}\left(k_{1} R\right) H_{m}^{(1) \prime}(k R)},
$$

where $J_{m}^{\prime}(x) \equiv(d / d x) J_{m}(x)$ and $H_{m}^{(1) \prime}(x) \equiv(d / d x) H_{m}^{(1)}(x)$.

We compute next the minimum convex source region of the far field radiation pattern defined in (9) and (30). For this purpose we use Main Result 1. Using (20) and (26) and Stirling's formula

$$
\Gamma(m+1) \sim \sqrt{2 \pi m}\left(\frac{m}{e}\right)^{m}, \quad m \longrightarrow \infty,
$$

we get from (30) that

$$
\begin{aligned}
\lim _{m \rightarrow \infty} \Gamma(m)\left(\frac{2}{k b}\right)^{m}\left|a_{m}\right| \\
=C \lim _{m \rightarrow \infty} \frac{\left(k R^{2} / 2 b\right)^{m}}{\Gamma(m+1)} \\
=0 \quad b>0,
\end{aligned}
$$

where

$$
\begin{gathered}
C=\pi\left|\frac{1-\sqrt{\epsilon_{r} / \mu_{r}} k / k_{1}}{1+\sqrt{\epsilon_{r} / \mu_{r}} k / k_{1}}\right|, \\
\lim _{m \rightarrow \infty} \Gamma(m)\left(\frac{2}{k b}\right)^{m}\left|a_{m}\right|=\infty \quad b=0 .
\end{gathered}
$$

It follows from these results and Main Result 1 that the minimum source region of the far field scattering amplitude associated with this scattered field is a point, in particular, the center of the circular cylinder (corresponding to $\rho=0$ ) that scatters the incident plane wave. This is a somewhat surprising result. It indicates that the center of the scatterer can be deduced unequivocally from the far field, but the information about the size of the scatterer is very limited since a very small source could produce the same far field, at least in theory (disregarding practical physical limitations of small antennas). Before we discuss its interpretation, which will naturally lead us to a more practical numerical computation counterpart of the theory and algorithms considered up to this point, we wish to briefly consider a variant of the scattering cylinder above and a generalization of the results above to near field incident wave excitation, which will prove to be quite insightful.

3.2. Scattering by a Conducting Circular Cylinder. In particular, for the case of a circular cylinder made of a perfect electric conductor (PEC) we have that the scattered field $E_{z}$ is given by (29) with the multipole moments given by ([14], page 604)

$$
a_{m}=-i^{m} \frac{J_{m}(k R)}{H_{m}^{(1)}(k R)}
$$

and by manipulations analogous to those leading to (32) we again obtain

$$
\lim _{m \rightarrow \infty} \Gamma(m)\left(\frac{2}{k b}\right)^{m}\left|a_{m}\right|=0 \quad b>0,
$$

which means that, as in the dielectric case, the minimum source region of the far field scattering amplitude associated with the field scattered by a PEC circular cylinder upon plane wave incidence is simply the center of the cylinder.

3.3. Line Source Scattering by a PEC Circular Cylinder. In the previous two examples we assumed that the incident wave is a plane wave. Next, we generalize the results by considering the tractable case of scattering of the field due to an infinitely long antenna (in the respective $3 \mathrm{D}$ picture) or a point source located outside the PEC circle (in the equivalent 2D framework). In particular, the field scattered by the PEC cylinder upon incidence of the field, due to an infinite line of constant current $I_{e}$ located at position $\mathbf{r}^{\prime}$ given in cylindrical coordinates as $\mathbf{r}^{\prime}=\left(\rho^{\prime}, \phi^{\prime}\right)$, is given by ([14], section 11.5.5)

$$
E_{z}(\rho, \phi)=-\frac{k^{2} I_{e}}{4 \omega \epsilon} \sum_{m=-\infty}^{\infty} a_{m} H_{m}^{(1)}(k \rho) e^{-i m \phi} \quad \rho>R,
$$

where

$$
a_{m}=-\frac{J_{m}(k R)}{H_{m}^{(1)}(k R)} H_{m}^{(1)}\left(k \rho^{\prime}\right) e^{i m \phi^{\prime}}
$$

so that

$$
\lim _{m \rightarrow \infty} \Gamma(m)\left(\frac{2}{k b}\right)^{m}\left|a_{m}\right|=\lim _{m \rightarrow \infty} \frac{\Gamma(m)\left(R^{2} / b \rho^{\prime}\right)^{m}}{\Gamma(m+1)}
$$


which means

$$
\begin{array}{ll}
\lim _{m \rightarrow \infty} \Gamma(m)\left(\frac{2}{k b}\right)^{m}\left|a_{m}\right|=0 & b>\frac{R^{2}}{\rho^{\prime}}, \\
\lim _{m \rightarrow \infty} \Gamma(m)\left(\frac{2}{k b}\right)^{m}\left|a_{m}\right|=\infty & b<\frac{R^{2}}{\rho^{\prime}} .
\end{array}
$$

In view of Main Result 1, this means that the smallest ball circumscribing the minimum source region is the ball $B_{\min }=$ $\left\{\mathbf{r} \in \mathbb{R}^{2}: \rho \leq R^{2} / \rho^{\prime}\right\}$. In other words, the largest dimension $D$ of the minimum source region must be $D=2 R^{2} / \rho^{\prime}$. Note that if $\rho^{\prime} \rightarrow \infty$ as is the case for the plane wave excitation of the previous section, we obtain $D=0$ corresponding to a multipole source localized in the origin as we found in the previous section.

3.4. Line Source Scattering by a Dielectric Circular Cylinder. The near field counterpart of the dielectric scattering by the plane wave considered above is the dielectric scattering under line source excitation. It follows from basic scattering theory ([14, chapter 11]; see, e.g., [15, equations (77)-(79)]) that the $z$-component of the scattered field due to the line source at $\left(\rho^{\prime}, \phi^{\prime}\right)$ is given by

$$
E_{z}(\rho, \phi)=-\frac{\omega \mu}{4} \sum_{m=-\infty}^{\infty} a_{m} H_{m}^{(1)}(k \rho) e^{-i m \phi} \quad \rho>R,
$$

where

$$
\begin{aligned}
a_{m}= & H_{m}^{(1)}\left(k \rho^{\prime}\right) e^{i m \phi^{\prime}} \\
& \times \frac{J_{m}^{\prime}(k R) J_{m}\left(k_{1} R\right)-\sqrt{\epsilon_{r} / \mu_{r}} J_{m}(k R) J_{m}^{\prime}\left(k_{1} R\right)}{\sqrt{\epsilon_{r} / \mu_{r}} H_{m}^{(1)}(k R) J_{m}^{\prime}\left(k_{1} R\right)-H_{m}^{(1)^{\prime}}(k R) J_{m}\left(k_{1} R\right)}
\end{aligned}
$$

which implies

$$
\lim _{m \rightarrow \infty} \Gamma(m)\left(\frac{2}{k b}\right)^{m}\left|a_{m}\right|=\frac{C}{\pi} \lim _{m \rightarrow \infty} \frac{\Gamma(m)\left(2 R^{2} / b \rho^{\prime}\right)^{m}}{\Gamma(m+1)}
$$

which means

$$
\begin{array}{ll}
\lim _{m \rightarrow \infty} \Gamma(m)\left(\frac{2}{k b}\right)^{m}\left|a_{m}\right|=0 & b>\frac{R^{2}}{\rho^{\prime}}, \\
\lim _{m \rightarrow \infty} \Gamma(m)\left(\frac{2}{k b}\right)^{m}\left|a_{m}\right|=\infty & b<\frac{R^{2}}{\rho^{\prime}} .
\end{array}
$$

This is the same result found in (38) and (39) for the PEC case. Thus we have found that, for both PEC and dielectric circular cylinders, the smallest ball circumscribing the minimum source region is the ball $B_{\min }=\left\{\mathbf{r} \in \mathbb{R}^{2}: \rho \leq R^{2} / \rho^{\prime}\right\}$. We elaborate and interpret further next in connection with the concept of UWSC sets predicted in [4].

3.5. Example of the Union of Well-Separated Convex Sets. The result above gives a first estimate of the minimum
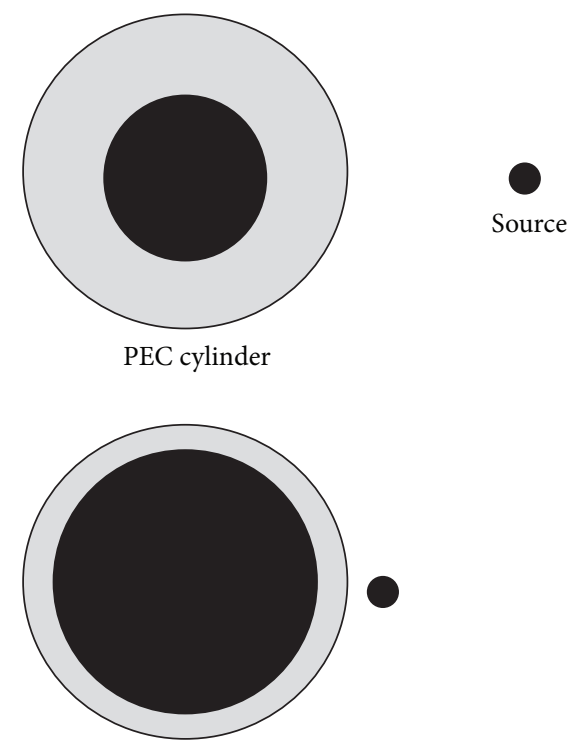

FIGURE 5: Estimated UWSC sets (shown in black), for the total far field due to a source (infinite line source in 3D space or equivalently point source in the $2 \mathrm{D}$ formulation) and a circular cylinder (PEC or dielectric) in its vicinity. The estimate for the minimum convex source region of the scatterer source depends on the proximity to the radiating source. The closer it is, then the larger the minimum source region needs to be in order to be able to radiate the corresponding scattering field.

convex source region of the scatterer. Furthermore, the field due to the combined line source and scatterer is the linear superposition of the fields of the line source and the source induced in the scatterer. In $2 \mathrm{D}$ space the minimum source region of the line source is the single point $\mathbf{r}^{\prime}=\left(\rho^{\prime}, \phi^{\prime}\right)$, where the source is located. The field due to the point source cannot be annulled by that of the scatterer source since it does not fully cover (enclose) the point source. In particular, it is not hard to show via Green's theorem that this field and the one due to the scatterer are linearly independent. Therefore the minimum source region of the field due to both is the union of the minimum source regions of the point source and the scatterer source individually. It follows that we have an estimate for the minimum source region of this total field as given by the union of the convex set $B_{\text {min }}=\left\{\mathbf{r} \in \mathbb{R}^{2}: \rho \leq\right.$ $\left.R^{2} / \rho^{\prime}\right\}$ and the single point $\mathbf{r}^{\prime}=\left(\rho^{\prime}, \phi^{\prime}\right)$. This is an exciting example of the existence and analytical computation of the UWSC sets of a far field whose existence was predicted in [4]. This result is illustrated in Figure 5.

3.6. Infinite-versus Finite-Dimensional Approaches. The analytical inverse support results above establish a fundamental constraint on the support of any source that produces the given scattered field. In the case of the plane wave excitation, which corresponds also to the limit $\rho^{\prime} \rightarrow \infty$, we found that the analytical minimum source region is the center of the scattering cylinder, a result that is quite surprising. On the other hand, for excitation by a line source at $\left(\rho^{\prime}, \phi^{\prime}\right)$ we found that, for both PEC and dielectric cylinders, the minimum 
spherical source region has the finite radius $R^{2} / \rho^{\prime}$. The larger minimum source region can be thought of as representing greater information about the source support that is accessible in the far field when the scatterer is excited by a near field source, which intuitively makes sense. These results establish bounds for the true scatterer support. In the present case they define for far field excitation the center of the scatterer and for near field probing a larger region which must be in the interior of the actual scatterer responsible for the given scattered field data. It is also important to emphasize that these results apply to the infinite-dimensional multipole domain and are thus relevant only for perfect far field data. For a source whose radius is as large as the predicted minimum radius, the terms in the infinite series expansion for the source energy decay sufficiently fast at infinity, so that the resulting source energy is not infinite; however, it can be prohibitively large. Thus strictly speaking, the source energy is not infinite; however, it can be quite large for a source in the predicted minimum region. Furthermore, it can be shown that regardless of $\rho^{\prime}$ the number of essential dimensions of the multipole domain is governed by the ruleof-thumb formula $a_{m} \simeq 0, m>k R$, so that, by plotting $a_{m}$ versus $m$ and estimating the knee of the plot, one can approximately estimate via this rule-of-thumb formula the circular cylinder's radius. We experimented with this finitedimensional approach and found it to be quite robust.

\section{Numerical Illustration}

The numerical counterpart of Main Results 1 and 2 employs finite-dimensional field data and a finite number of test origins. For a reference origin, for example, we compute the far field radiation pattern and the corresponding multipole moments via (10). We keep only the nonnegligible multipole moments and take the rest to be zero. We found that, in the examples to be discussed in the following, the number of significant multipole moments corresponds approximately to the truncation $a_{m} \simeq 0, m>k R$, where $R$ is the radius of the smallest circle that circumscribes the original source support and is centered at the same origin. This step makes the far field pattern and multipole moment data finite-dimensional for the remaining computations. Main Results 1 and 2 are implemented requiring that the functional energy of the reconstructed field, as defined in (18) and (24), exhibit a reasonable finite value. We experimented with several ways of implementing this cutoff and settled in an approach resembling the familiar L-curve method. In particular, we automatically estimated the peak curvature point of the "log(energy) versus radius" curve and used this peak curvature point as the numerical estimate of the minimum radius $R_{\min }$ in the convex support estimation (Main Result 1) or of $R_{\min }^{\text {int }}$ in the complementary nonconvex support estimation (Main Result 2). Next we discuss this computational implementation with two examples. The first illustrates the use of support information in an inverse source problem. The second sheds light on the numerical counterpart of the scattering PEC cylinder case discussed in Section 3.

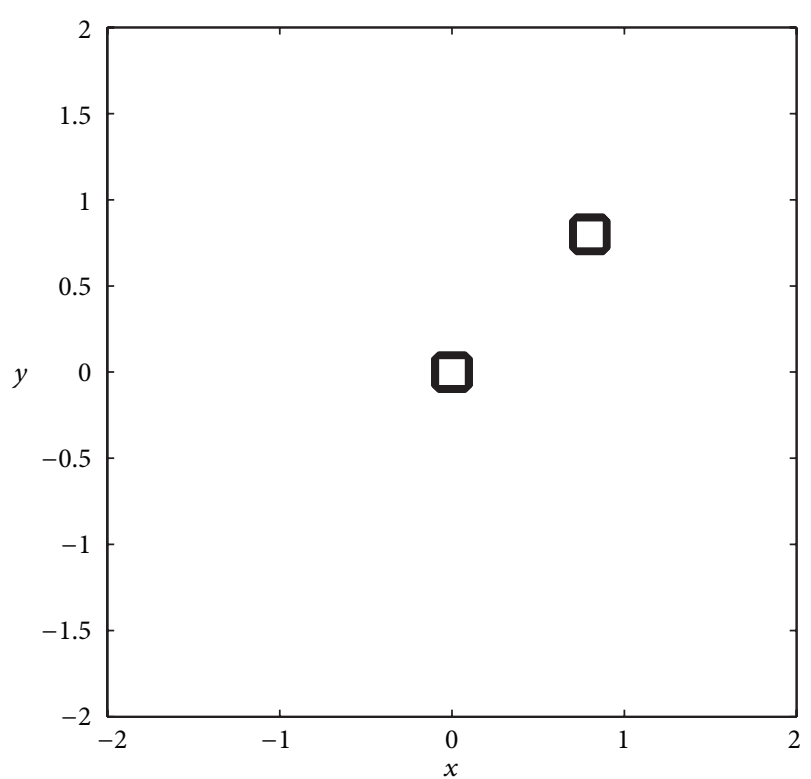

FIGURE 6: Contour plot of the unit-amplitude source used in the simulations.

4.1. Inverse Source Problem with Support Estimation. As a first numerical example, we consider the inverse source problem of estimating an unknown radiating source from knowledge of the far field radiation pattern it generates. As part of the inversion, we incorporate estimation of the minimum source region consistent with the given far field. The support information is used as prior for the reconstruction of the minimum energy source that resides in that support and gives the desired far field data. To estimate the minimum source region, we focus first on the estimation of a convex bound for the minimum source region. Later we refine the estimate of the minimum source region up to a nonconvex region, via the methodology presented in the paper. The final result is that the reconstructed source is quite close to the original source generating the far field data, as desired.

Figure 6 shows the source used in the simulations. It is formed by two $0.2 \lambda \times 0.2 \lambda$ squares, where $\lambda$ is the wavelength. The total source is contained inside a $\lambda \times \lambda$ square and thus has maximal dimension $\sqrt{2} \lambda$. In the following all dimensions are given as multiples of $\lambda$. The source distribution inside the squares is uniform and has unit amplitude. Figure 7 shows the polar diagram of the amplitude of the far field radiation pattern generated by this source. First, some insight about the source to be reconstructed and its near field can be gained by backpropagating the far field to the near zone. Figure 8 illustrates this backpropagated field. The peaks in this plot offer qualitative insight about the location and size of the two square sources composing the total source. On the other hand, the near field associated with this backpropagation is not the correct near field (as we show next). Furthermore, the value of the source cannot be deduced from this qualitative insight alone. In the following, we implement the full reconstruction of the source by means of a three-step procedure based on the methods developed in this paper. 


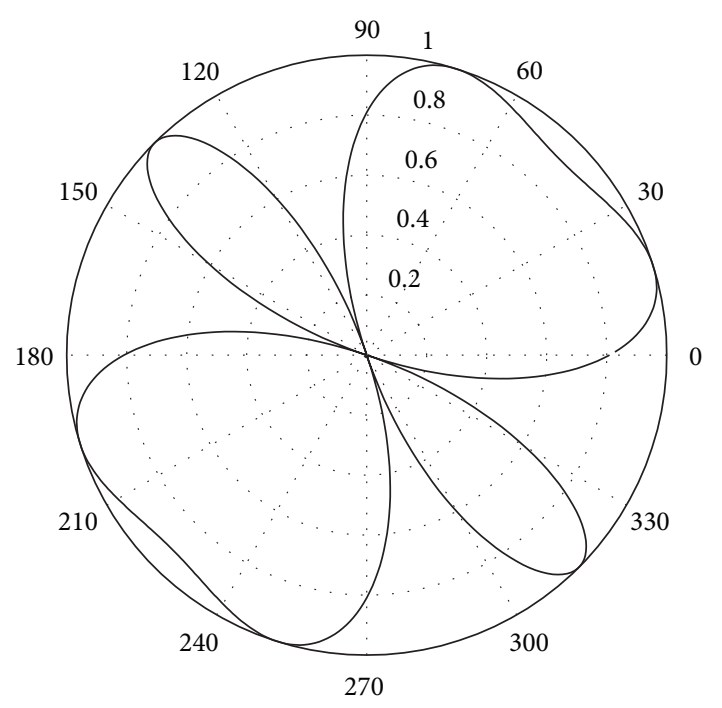

FIgURE 7: Polar diagram of the radiation pattern amplitude.

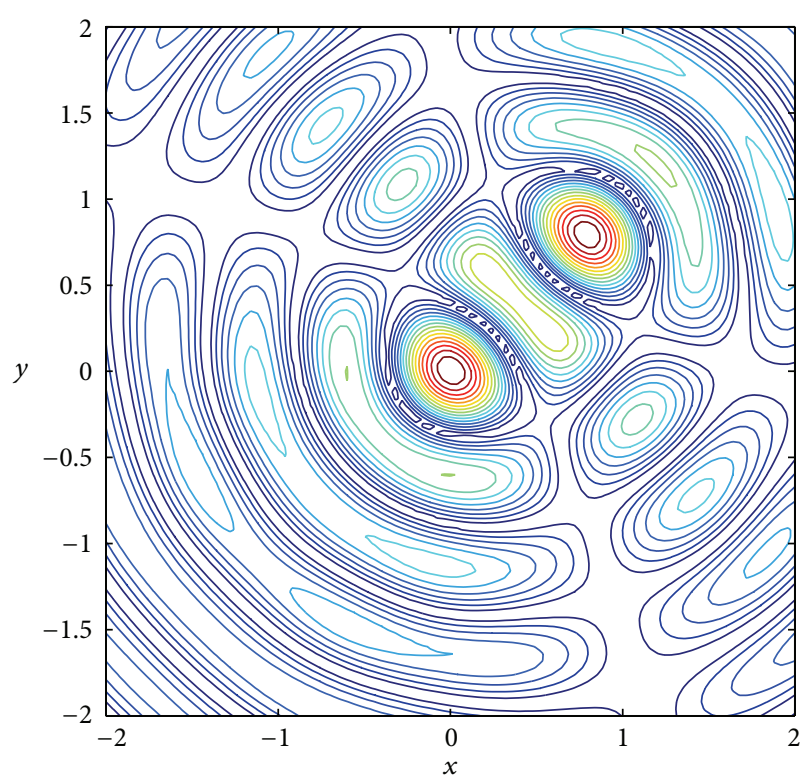

FIGURE 8: Contour plot of the backpropagated field amplitude.

First we estimate the minimum convex source region and compute the minimum energy source associated with this estimated region. Next we compute the near field based on the reconstructed minimum energy source and use this information to obtain a tighter, nonconvex estimate of the minimum source region. Finally, we compute the minimum energy source associated with this nonconvex estimate. The thus reconstructed source is very similar to the original source used in generating the far field data, as desired.

To estimate the minimum convex source region, we considered 6 test origins: $(-10,0),(10,0),(0,-10),(0,10)$, $(-10,10)$, and $(10,-10)$. Figure 9 shows the computed estimate of the minimum convex source region obtained using these test origins. Also shown in the figure is the true source support, which is contained inside the convex source

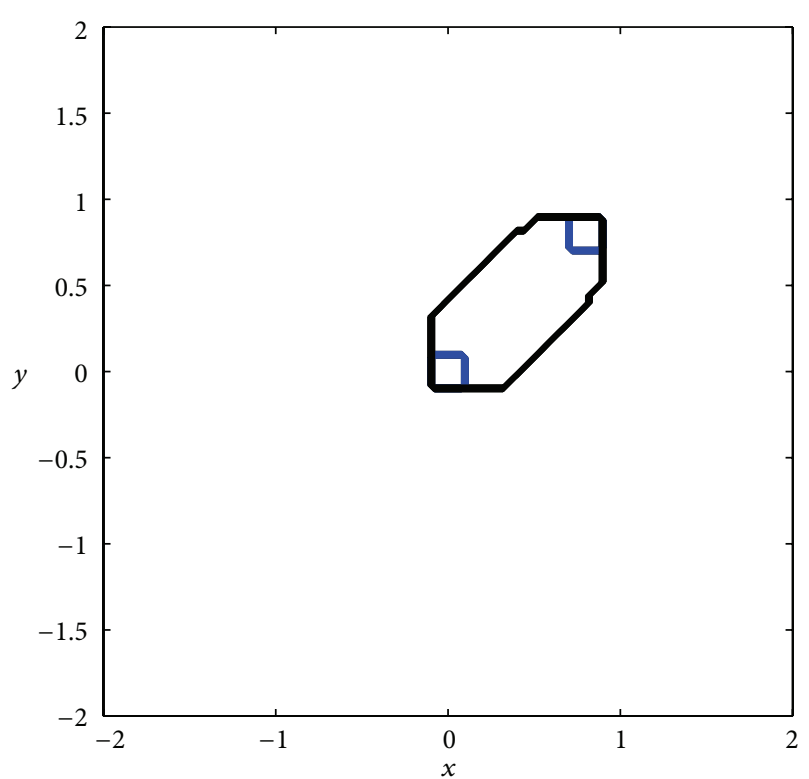

FIGURE 9: Minimum convex source region estimate computed with test origins $(-10,0),(10,0),(0,-10),(0,10),(-10,10)$, and $(10,-10)$. Also shown is the true source support, which is contained inside this region.

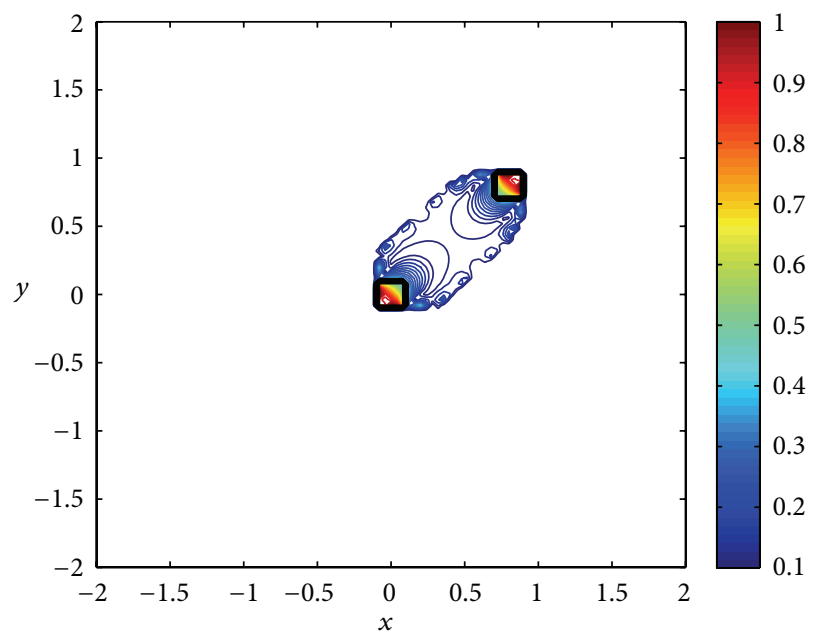

Figure 10: Contour plot of the minimum energy source contained in the minimum convex source region estimate corresponding to test origins $(-10,0),(10,0),(0,-10),(0,10),(-10,10)$, and $(10,-10)$. The high amplitude region of this source (value near 1$)$ coincides very well with the correct source support of the original unitamplitude source, as desired.

region estimate, as expected. Using this prior source support information, we computed next the minimum energy source contained in this source region that is consistent with the far field data. This is done by standard matrix inversion giving the solution of minimum 2 norm to the inverse problem of finding a source in the given support that produces the given data. The contour plot of the reconstructed source is given in Figure 10. The source regions where the amplitude 


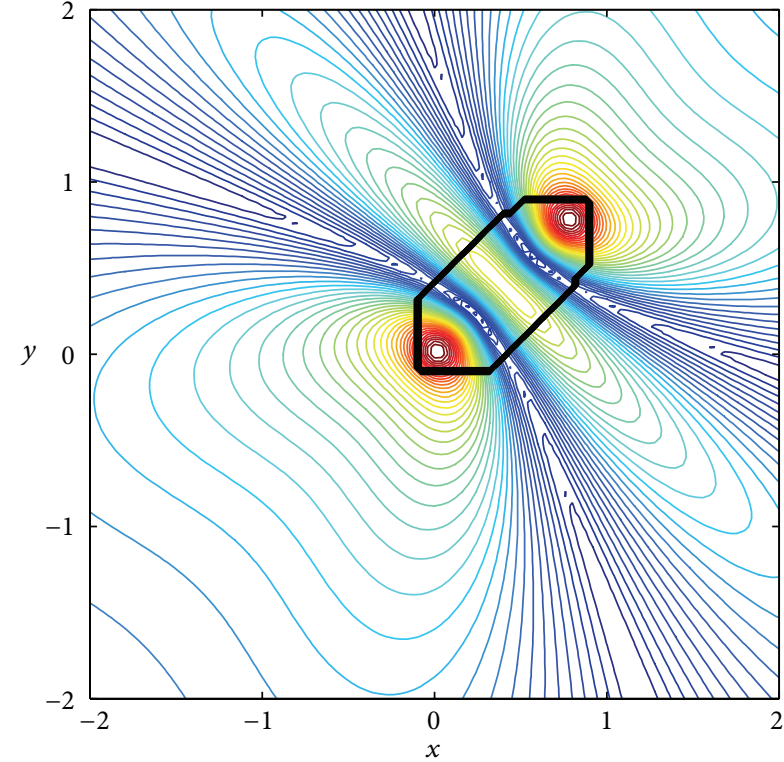

(a)

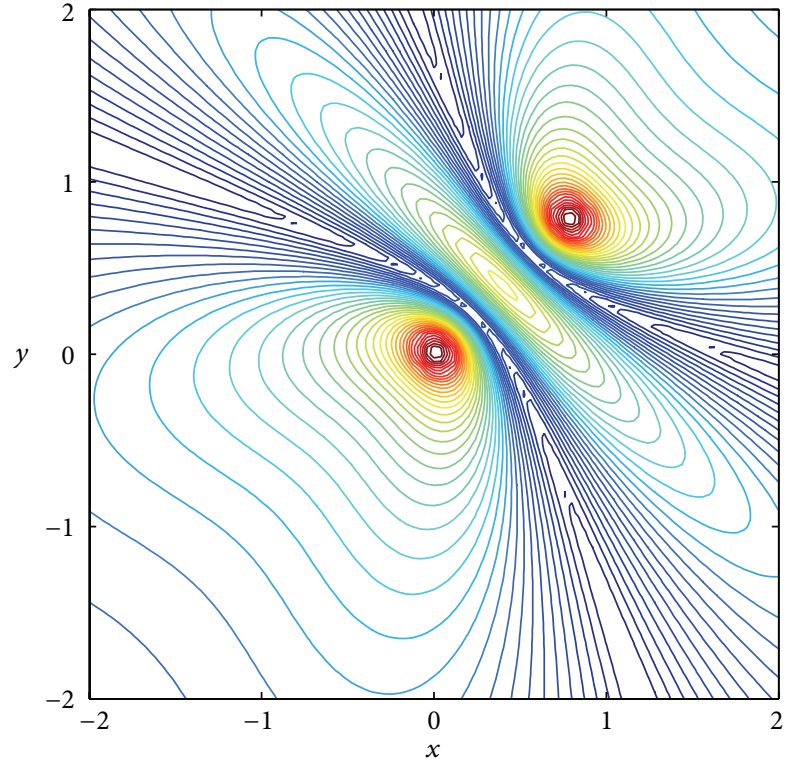

(b)

FIgURE 11: (a) Contour plot of the magnitude of the near field radiated by the minimum energy source. The support of this source is the minimum convex source region estimate corresponding to test origins $(-10,0),(10,0),(0,-10),(0,10),(-10,10)$, and $(10,-10)($ shown in black color). (b) Contour plot of the magnitude of the near field radiated by the original source. The two plots are almost identical, as desired.

is significant (close to 1) are seen to coincide quite well with the true source support. Furthermore, this step also allows us to estimate the near field, by computing the radiation associated with the reconstructed minimum energy source. Figure 11 illustrates the near field associated with this source. The figure shows both the computed near field and the correct near field based on the original source, and the two coincide very well, as desired. This estimate of the near field is used in the following step, which seeks to refine the estimate of the minimum source region, up to its possibly nonconvex nature. Using the estimated near field, we implemented the method presented in the paper to extract further information about the possibly nonconvex minimum source region. Using two test origins, $(1.2,-.3)$ and $(-.3,1.2)$, we obtained the better, nonconvex estimate of the minimum source region shown in Figure 12. As a final step in the reconstruction, we did the standard linear inversion leading to the minimum energy source that is consistent with the far field data and is confined within this better estimate of the minimum source region. The reconstructed source is shown in Figure 13. The source strength is clearly focused on the vicinity of the true source support, and the value of the source is near unity in this region, as desired. Thus this three-step methodology, exploiting the extracted source support information via the multipole inverse theory established in the paper, is quite practical, as well as insightful about the possibilities and limitations of inverse source problems with far field data. For comparison purposes, we present in Figure 14 a plot of the minimum energy source that would have been obtained in the same computational grid if no prior information about the source support would have been sought during the inversion process. Note that this source is quite similar to the backpropagated field in Figure 8 (which is not surprising in view of the multipole expansion). The reconstruction in Figure 13, which seeks and exploits support information from far field data, is clearly superior.

4.2. Numerical Scattering Example. As a second numerical case, we considered the numerical computations associated with the scattering PEC cylinder discussed in Section 3. First we addressed the estimation, from the far field, of the minimum convex source region associated with the source that is induced in a PEC cylinder due to different excitations. We found that very accurate estimates of the scatterer support can be obtained via the computational counterpart of Main Result 1 for scatterers whose radius $R$ is greater than about a couple of wavelengths. For smaller cylinders, the method gives excellent reconstruction of the section of the cylinder that is illuminated but gives a more ambiguous boundary for the nonilluminated portion of the cylinder, which cuts part of that half of the cylinder. A more interesting problem consists of estimating bounds for the total source support, including both the excitation point source and the scattering PEC cylinder. Next we focus on this problem, illustrating the application of the computational counterparts of Main Results 1 and 2. In the following calculations, we begin with the convex estimate of the minimum source region. Later we refine the bounding region for the minimum source region using the complementary nonconvex boundaries. We use a PEC cylinder of radius $R=5$ (where, as before, all dimensions are given as multiples of $\lambda$ ). We consider two particular cases: 


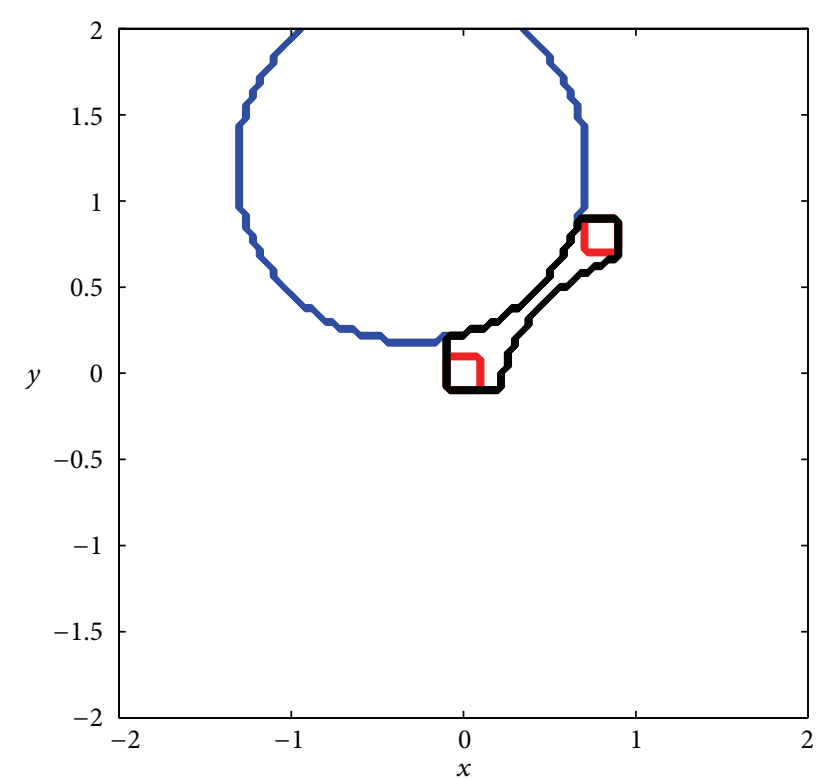

FIGURE 12: Estimated nonconvex region bounding the minimum source region. It was obtained using test origins $(1.2,-.3)$ and $(-.3,1.2)$. Also shown are the true source support and the boundary of the minimum source region associated with test origin $(-.3,1.2)$.

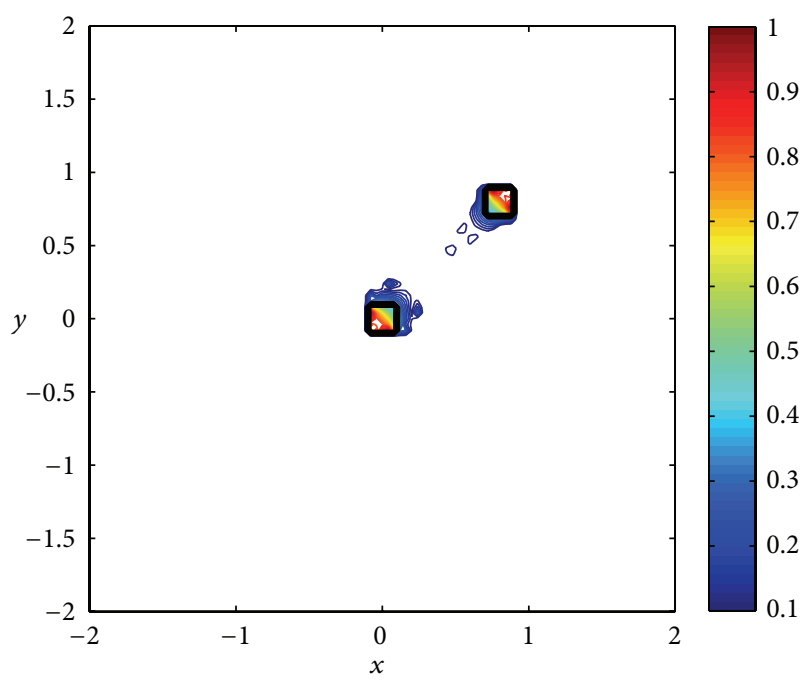

FIGURE 13: Reconstructed minimum energy source contained in the nonconvex estimate of the minimum source region. Also shown, in black color, is the support of the original unit-amplitude source. The reconstructed source approximates very well the original source, as desired.

(1) an excitation source at position $\left(x^{\prime}, y^{\prime}\right)=(-10,0)$ and (2) two excitation sources, at positions $(-15,-5)$ and $(15,-5)$.

For the case of a single excitation source at $(-10,0)$, we employed the following 4 test origins to obtain a first convex boundary for the total source (the point source plus the scatterer): $(-50,0),(50,0),(10,50)$, and $(10,-50)$. Figure 15 shows the computational sequence corresponding to these test origins. The plots show the correct source support, the estimated boundary of the minimum circle of the current

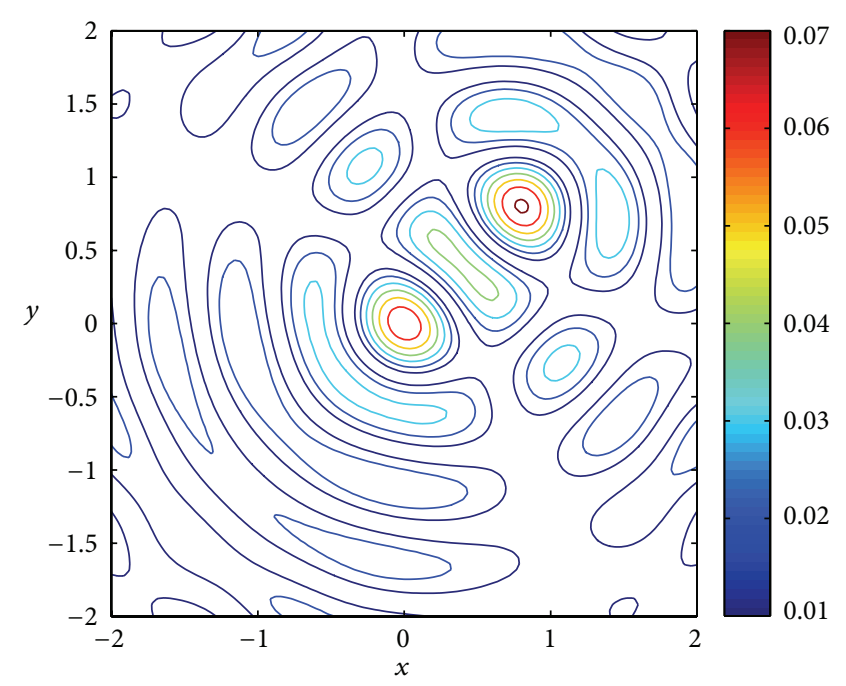

FIgUre 14: Amplitude of the minimum energy solution to the inverse source problem without support information.

computational step, and the estimated boundary of the convex source region up to the given step. Figure 16 shows the cummulative convex support estimate after adding two more test origins, at $(-50,-50)$ and $(-50,50)$. The results based on the computational form of Main Result 2 are illustrated in Figure 17, for test origin $(-10,11)$. Finally, Figure 18 shows a final refinement of the boundary of the minimum source region based on the test origins above, plus two more test origins (at $(30,50)$ and $(30,-50))$ for the convex part and three more test origins (at $(-10,-11),(-10,8)$, and $(-10,-8)$ ) for the nonconvex part. We tried other origins but concluded that only marginal support information was available beyond this point. The nonconvex part, in particular, could not be used to isolate the supports of the point source and the scatterer.

Figure 19 illustrates the convex support estimation results for the case of two point sources illuminating the PEC cylinder. The convex support shown in the figure corresponds to 6 test origins: $(0,-50),(0,50),(-20,-50),(20,-50),(-50,0)$, and $(50,0)$. Figure 20 shows the nonconvex support estimate based on 5 test origins: $(-14,9),(14,9),(0,-15),(-7,-12)$, and $(7,-12)$.

\section{Conclusion}

We developed a theoretical and computational framework to exactly or approximately estimate the minimum source region of a far field. The methodology constitutes the multipole theory counterpart of the plane wave expansion approach of a previous paper [2]. The proposed method allows handling of problems for which the far field is given as a multipole series expansion (e.g., cylindrical scatterers), for which the method in [2] cannot be used since it requires knowledge of the far field pattern versus the observation direction in closed form. We found that, for canonical cylinders and under plane wave excitation, the analytical results define only the center of the scatterer. On 


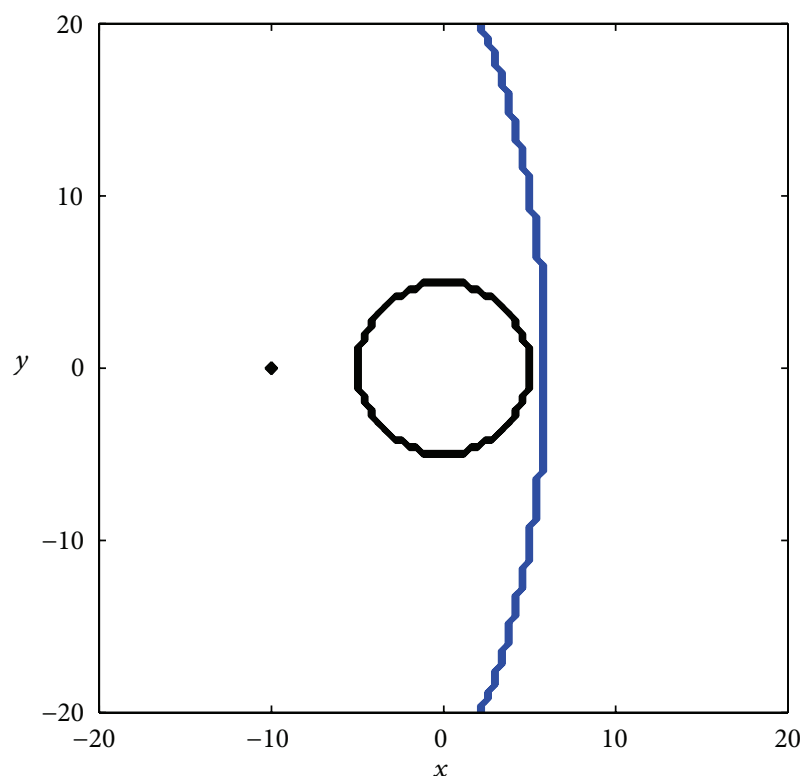

(a)

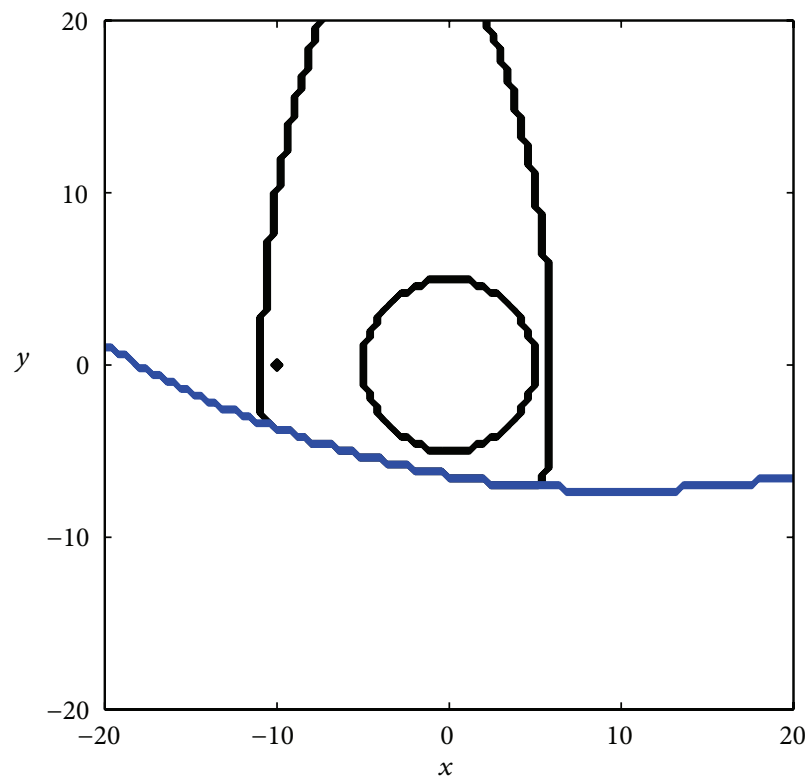

(c)

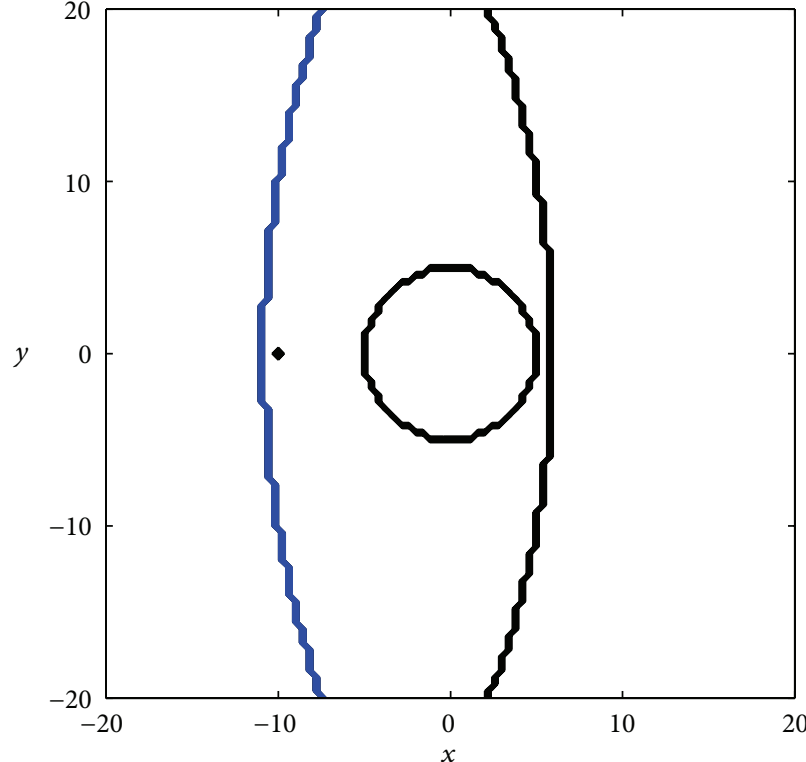

(b)

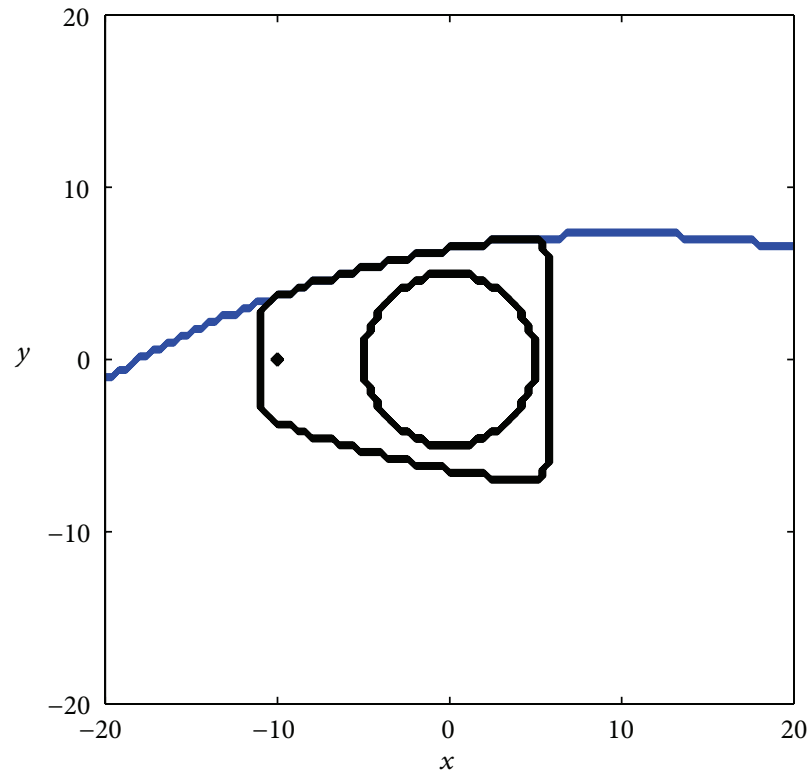

(d)

FIGURE 15: Minimum circles and cummulative estimated convex boundary of the minimum source region for 4 test origins: $(-50,0)(a)$, $(50,0)(b),(10,50)(c)$, and $(10,-50)(d)$. Also shown is the correct support of the total source, formed by the point source and the scattering PEC cylinder.

the other hand, under near field excitation, the boundary of the minimum source region associated with the induced source in the scatterer is a circle of finite radius inside the scatterer. This can be thought of as rigorous quantification of the greater information about the scatterer available with near field excitation. Furthermore, due to reciprocity, the role of the (near field) excitation and the (far field) receiver can be interchanged, so that the same results also apply for plane wave excitation (far field excitation) and sensing of the scattered field in the near zone. Thus the same results, with this role reversal, coincidentally show that the same developments derived in the paper can be applied to the companion problem of estimating minimum source regions from near field data. This, in turn, further demonstrates the broad applicability of the ideas about minimum source regions considered in this paper and in previous work.

The analytical results can be thought of as a quantification of the scatterer support information that is inferrable from the far scattered field. They show that this information is, in theory, critically affected by the proximity of the excitation 


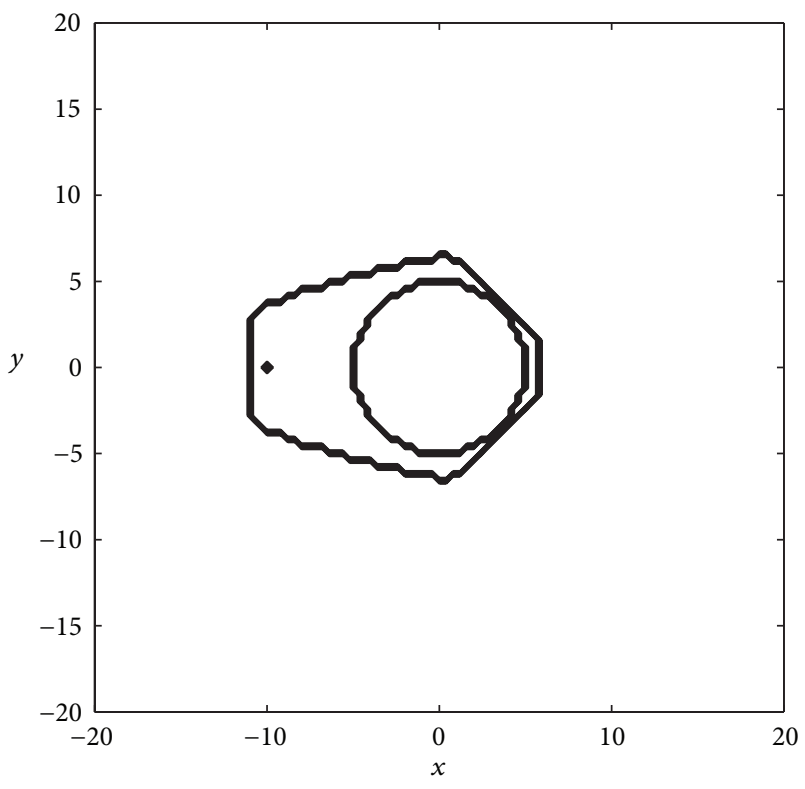

FIGURE 16: Cummulative estimated convex support for 6 test origins: $(-50,0),(50,0),(10,50),(10,-50),(-50,-50)$, and $(-50,50)$.

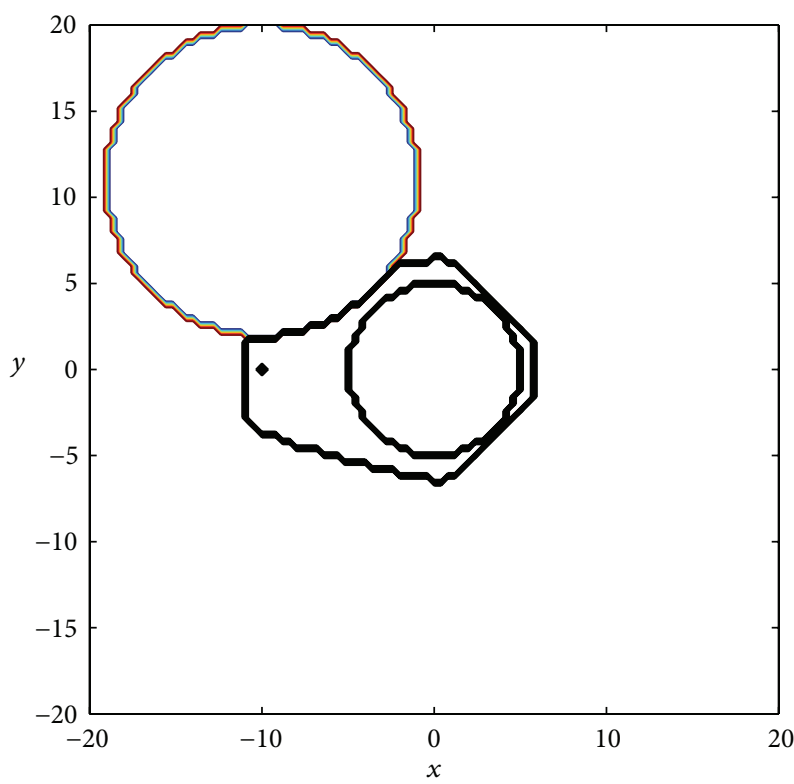

Figure 17: Cummulative estimated nonconvex support, based on the estimated circular boundary (shown in the figure) corresponding to test origin $(-10,11)$.

source to the scatterer. The provided analytical results also illustrate the UWSC sets proposed in a previous paper. The analytical results are fundamental (they are strict bounds to the minimum source regions), but at least in the particular case of the PEC and dielectric cylindrical scatterers considered in the paper they are also quite restrictive if the excitation source is far from the scatterer (the minimum source region becomes very small as $\rho^{\prime}$ becomes larger, relative to the support of the true scatterer responsible for

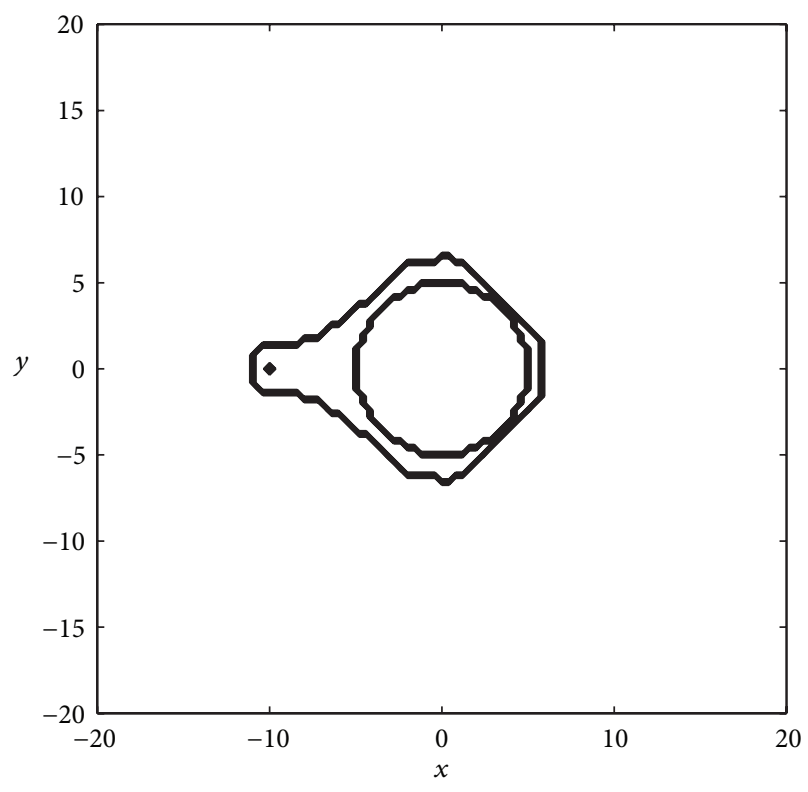

FIGURE 18: Cummulative estimated nonconvex support based on 8 test origins for the convex part and 4 test origins for the nonconvex part.

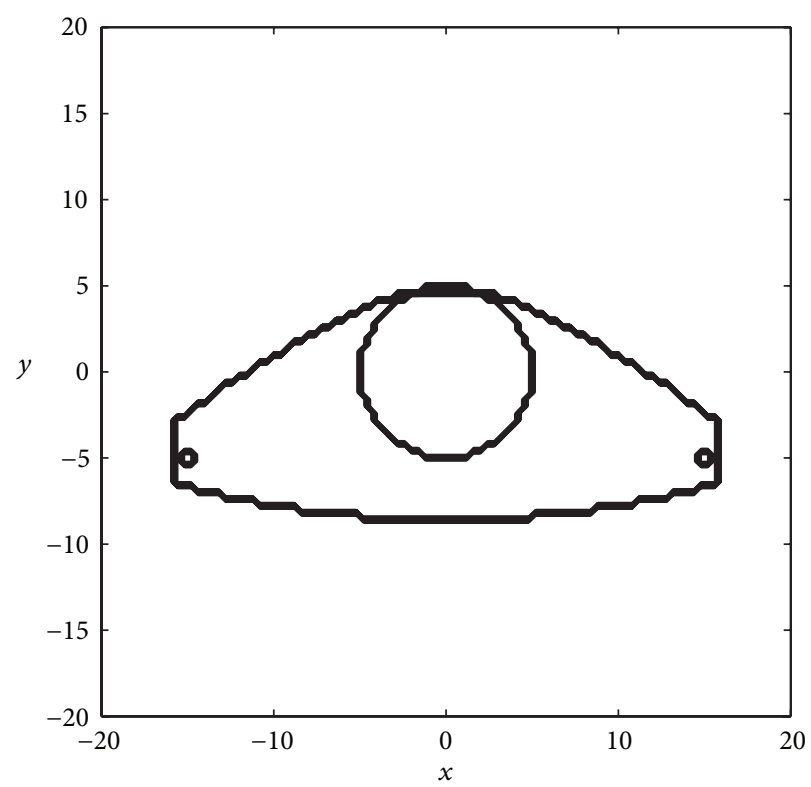

FIGURE 19: Cummulative estimated convex support for test origins $(0,-50),(0,50),(-20,-50),(20,-50),(-50,0)$, and $(50,0)$.

the data). This limitation naturally led us to the more practical computational implementation of the ideas derived in the paper, where the field data are essentially finite-dimensional and the realizability constraint becomes a bounding of the functional energy. This regularization led to results such as those shown in Section 4, which are more conservative but also more stable.

The provided analytical and numerical inverse support results based on the methods derived in the paper were quite 


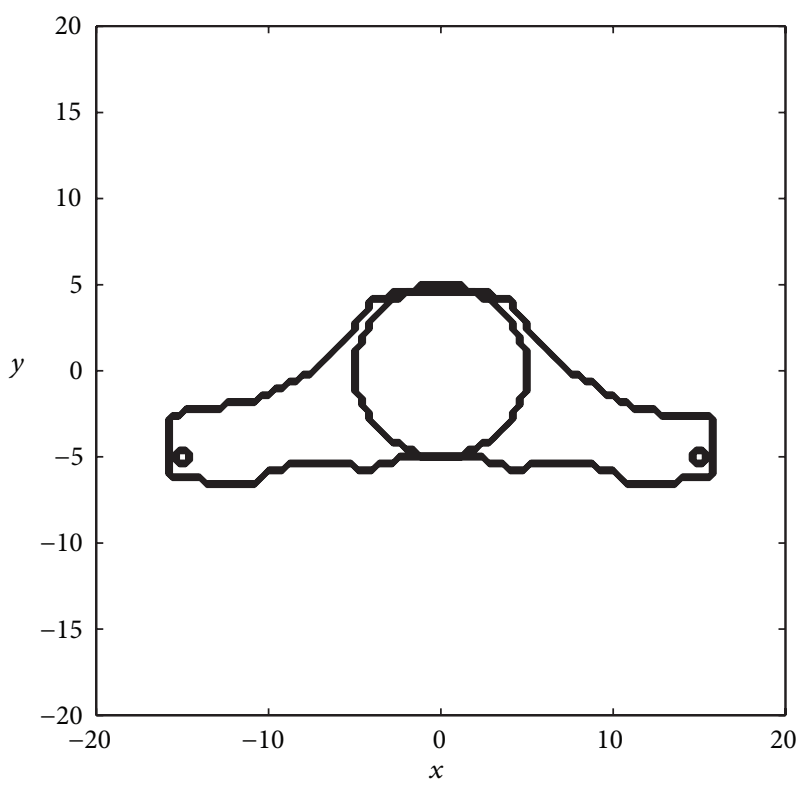

FIGURE 20: Cummulative estimated nonconvex support based on 6 test origins for the convex part and 5 test origins for the nonconvex part.

encouraging. Thus the proposed methods are useful for the particular sources we considered, and we suspect that they work for a broad class of sources. We acknowledge, however, that the nonconvex bounds may not be correct for some sources. We have not specified in this paper the class of sources for which these methods (the nonconvex bounds, in particular) are expected to be applicable in practice. This pending question is related to issues raised in [4] and is left for the future.

We also wish to comment that, since the estimation of the near field from the far field is ill-posed, the nonconvex support estimation component of the proposed methodology (which relies on such near field estimation) is clearly ill-posed as well. Yet we have established the basic inverse-diffractionbased construction procedure which shows that it is theoretically possible to compute the possibly nonconvex minimum source region. The proposed methodology complements the previous efforts in this area which had emphasized only the minimum nonconvex source region. Furthermore, being aware of the practical challenge of the required near field estimation, we have examined the practical applicability of the proposed technique in the context of two numerical examples. In both cases we were able to extract some nonconvex support information, as desired. The usefulness of the extra nonconvex support information was illustrated in the numerical example related to the inverse source problem, where we were able to obtain a solid reconstruction of the source thanks to the nonconvex support estimation component of the proposed methodology.

We are currently working on a purely computational variant of the methods derived in this paper, which employs manifolds of realizable field data, corresponding to bounded functional energy sources. This approach offers insight onto the observed robustness of minimum source regions in inverse scattering formulations (as shown in the numerical simulations of this paper and those of other studies). We are also exploring the possibility of extending the derived methods to characterize and compute minimum source regions in inverse problems with partially correlated fields which arise in optical coherence theory. Finally, we have considered here the scalar formulation. Extension to the full vector electromagnetic fields, based on the corresponding vector multipole fields, appears at first sight equally tractable and provides another important avenue for future research. We plan to report our research findings in these and related areas elsewhere.

\section{Appendices}

\section{A. Proof of Main Result 1}

We provide the proof of Main Result 1 (21). The region of convergence may be closed or open. This explain the use of "inf" (infimum) in (19) instead of just "minimum." If it is closed, then

$$
\sum_{m=-\infty}^{\infty}\left|a_{m}\right|^{2}\left|H_{m}^{(1)}(k b)\right|^{2}<\infty
$$

holds for radius $b \geq R_{\min }$, while if it is open (A.1) holds for radius $b>R_{\min }$.

Let us consider first the case of a closed region of convergence. According to the D'Alembert ratio test and using the asymptotics (20), convergence at $b=R_{\min }$ implies

$$
\lim _{m \rightarrow \infty} \frac{\left|a_{m+1}\right|^{2}\left|H_{m+1}^{(1)}(k b)\right|^{2}}{\left|a_{m}\right|^{2}\left|H_{m}^{(1)}(k b)\right|^{2}}=\lim _{m \rightarrow \infty} \frac{\left|a_{m+1}\right|^{2}}{\left|a_{m}\right|^{2}}\left(\frac{2 m}{k R_{\min }}\right)^{2} \leq 1
$$

while divergence for $b<R_{\min }$,

$$
\sum_{m=-\infty}^{\infty}\left|a_{m}\right|^{2}\left|H_{m}^{(1)}(k b)\right|^{2}=\infty \quad b<R_{\min }
$$

implies that

$$
\begin{aligned}
\lim _{m \rightarrow \infty} & \frac{\left|a_{m+1}\right|^{2}\left|H_{m+1}^{(1)}(k b)\right|^{2}}{\left|a_{m}\right|^{2}\left|H_{m}^{(1)}(k b)\right|^{2}} \\
& =\lim _{m \rightarrow \infty} \frac{\left|a_{m+1}\right|^{2}}{\left|a_{m}\right|^{2}}\left(\frac{2 m}{k b}\right)^{2} \geq 1 \quad b<R_{\text {min }} .
\end{aligned}
$$

Since in (A.4) $b$ can be arbitrarily close to $R_{\min }$ and the $(k b)^{2}$ factor depends continuously on $b$ then according to (A.2) and (A.4) necessarily

$$
\begin{aligned}
\lim _{m \rightarrow \infty} \frac{\left|a_{m+1}\right|^{2}\left|H_{m+1}^{(1)}\left(k R_{\text {min }}\right)\right|^{2}}{\left|a_{m}\right|^{2}\left|H_{m}^{(1)}\left(k R_{\text {min }}\right)\right|^{2}} \\
\quad=\lim _{m \rightarrow \infty} \frac{\left|a_{m+1}\right|^{2}}{\left|a_{m}\right|^{2}}\left(\frac{2 m}{k R_{\min }}\right)^{2}=1
\end{aligned}
$$


and furthermore

$$
\begin{aligned}
\lim _{m \rightarrow \infty} & \frac{\left|a_{m+1}\right|^{2}\left|H_{m+1}^{(1)}(k b)\right|^{2}}{\left|a_{m}\right|^{2}\left|H_{m}^{(1)}(k b)\right|^{2}} \\
& =\lim _{m \rightarrow \infty} \frac{\left|a_{m+1}\right|^{2}}{\left|a_{m}\right|^{2}}\left(\frac{2 m}{k b}\right)^{2}>1 \quad b<R_{\min }
\end{aligned}
$$

so that necessarily

$$
\lim _{m \rightarrow \infty}\left|a_{m}\right|^{2}\left|H_{m}^{(1)}(k b)\right|^{2} \neq 0 \quad b<R_{\min }
$$

which gives, along with (20), the desired result

$$
\begin{aligned}
\lim _{m \rightarrow \infty} & \left|a_{m}\right|\left|H_{m}^{(1)}(k b)\right| \\
& =\frac{1}{\pi} \lim _{m \rightarrow \infty} \Gamma(m)\left(\frac{2}{k b}\right)^{m}\left|a_{m}\right| \neq 0 \quad b<R_{\min } .
\end{aligned}
$$

If on the other hand the region of convergence is open then

$$
\begin{aligned}
\lim _{m \rightarrow \infty} \frac{\left|a_{m+1}\right|^{2}\left|H_{m+1}^{(1)}\left(k R_{\min }\right)\right|^{2}}{\left|a_{m}\right|^{2}\left|H_{m}^{(1)}\left(k R_{\min }\right)\right|^{2}} \\
\quad=\lim _{m \rightarrow \infty} \frac{\left|a_{m+1}\right|^{2}}{\left|a_{m}\right|^{2}}\left(\frac{2 m}{k b}\right)^{2} \geq 1
\end{aligned}
$$

which by considerations analogous to those employed above gives (A.6) and in turn (A.8). It follows that the condition in (A.8) can be used to define $R_{\min }$ as is done in (21).

\section{B. Analytical Calculation for Point Sources}

We apply Main Results 1 and 2 to compute the minimum convex source region and minimum source region of two point sources in $2 \mathrm{D}$ space. Extension to $N$ sources follows readily with the same ideas. The sources have positions $\mathbf{X}_{1}$ and $\mathbf{X}_{2}$ and complex-valued excitation strengths $\alpha_{1}$ and $\alpha_{2}$, respectively. We let $\mathbf{X}_{1}=\left(\rho_{1}, \phi_{1}\right)$ and $\mathbf{X}_{2}=\left(\rho_{2}, \phi_{2}\right)$. Without loss of generality $\rho_{1} \geq \rho_{2}$. We have

$$
I_{z}=\alpha_{1} \delta\left(\mathbf{r}-\mathbf{X}_{1}\right)+\alpha_{2} \delta\left(\mathbf{r}-\mathbf{X}_{2}\right)
$$

so that

$$
a_{m}=\alpha_{1} J_{m}\left(k \rho_{1}\right) e^{i m \phi_{1}}+\alpha_{2} J_{m}\left(k \rho_{2}\right) e^{i m \phi_{2}} .
$$

Then

$$
\begin{aligned}
\lim _{m \rightarrow \infty} & \Gamma(m)\left(\frac{2}{k b}\right)^{m}\left|a_{m}\right| \\
& \leq \lim _{m \rightarrow \infty} \frac{\sqrt{\pi} \Gamma(m)}{2 \Gamma(m+3 / 2)}\left|\alpha_{1} e^{i m \phi_{1}}+\alpha_{2} e^{i m \phi_{2}}\right|\left(\frac{\rho_{1}}{b}\right)^{m},
\end{aligned}
$$

where we used (26) and the triangle inequality. It follows from (B.3) that

$$
\lim _{m \rightarrow \infty} \Gamma(m)\left(\frac{2}{k b}\right)^{m}\left|a_{m}\right|=0 \quad b>\rho_{1} .
$$

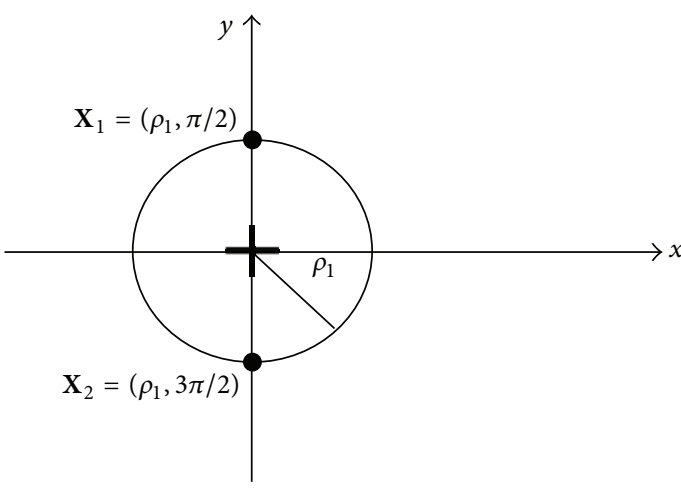

FIgURE 21: Minimum circular source region $B_{\min }$ of the far field of two point sources.

In addition, it is not hard to show via the reverse triangle inequality that

$$
\begin{aligned}
\lim _{m \rightarrow \infty} & \Gamma(m)\left(\frac{2}{k b}\right)^{m}\left|a_{m}\right| \\
& \geq \lim _{m \rightarrow \infty} \frac{\sqrt{\pi} \Gamma(m)}{2 \Gamma(m+3 / 2)}|| \alpha_{1}|-| \alpha_{2}||\left(\frac{\rho_{1}}{b}\right)^{m}
\end{aligned}
$$

which implies that

$$
\lim _{m \rightarrow \infty} \Gamma(m)\left(\frac{2}{k b}\right)^{m}\left|a_{m}\right|=\infty \quad b<\rho_{1} .
$$

It follows from (B.4) and (B.6) and Main Result 1 that $R_{\min }=$ $\rho_{1}$. Here we assumed $\rho_{1} \geq \rho_{2}$ so that more generally the result is $R_{\min }=\max \left(\rho_{1}, \rho_{2}\right)$. Here we considered two point sources, but it is not hard to extend these manipulations to $N$ point sources localized at $\mathbf{X}_{n}=\left(\rho_{n}, \phi_{n}\right), n=1,2, \ldots, N$, obtaining $R_{\text {min }}=\max \left(\rho_{1}, \rho_{2}, \ldots, \rho_{N}\right)$.

The result above defines the radius of the minimum circular source region $B_{\min }$ associated with a given origin. To define the minimum convex source region $B_{\text {min,conv }}$ we consider different origins. To fix ideas assume that a reference coordinate system is used such that $\mathbf{X}_{1}=\left(\rho_{1}, \pi / 2\right)$ and $\mathbf{X}_{2}=\left(\rho_{1}, 3 \pi / 2\right)$ (see Figure 21). For that origin we have $R_{\text {min }}=\rho_{1}$. The respective minimum circular source region is shown in Figure 21. If in a subsequent computation we adopt a new origin of coordinates whose position in the reference coordinate system is $O^{\prime}=\left(\rho_{0}, 0\right)$, then $R_{\min }=\sqrt{\rho_{0}^{2}+\rho_{1}^{2}}$. The associated minimum circle is shown in Figure 22. Note that this holds for any value of $\rho_{0}$. If we now take $O^{\prime \prime}=\left(\rho_{0}, \pi\right)$, we also get $R_{\min }=\sqrt{\rho_{0}^{2}+\rho_{1}^{2}}$. The respective minimum circular source region is shown in Figure 22. Since $\rho_{0}$ in $O^{\prime}$ and $O^{\prime \prime}$ is arbitrary, we can deduce the minimum convex source region from the behavior of the intersection of these minimum circular source regions as $\rho_{0} \rightarrow \infty$, which implies that it is the straight line defined by $\left\{(x, y) \in \mathbb{R}^{2}: x=0,|y| \leq \rho_{1}\right\}$ which unites the two source points, as shown pictorially in Figure 23. The respective generalization for $N$ point sources is that the minimum convex source region is the union of all the polygons whose vertices are the source points. 


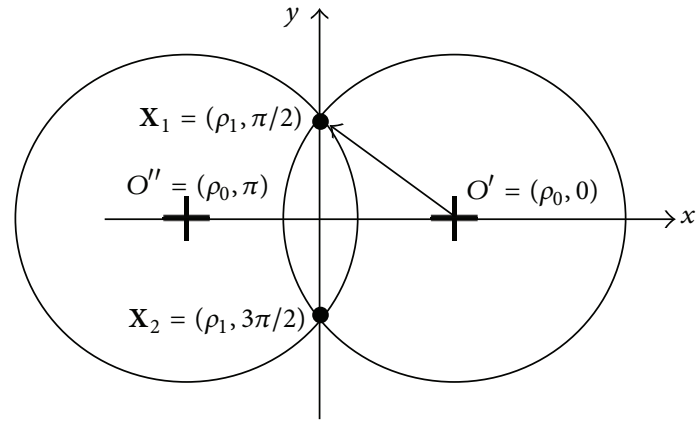

FIGURE 22: Minimum circular source regions $B_{\min }\left(O^{\prime}\right)$ and $B_{\min }\left(O^{\prime \prime}\right)$ of the far field of two point sources.

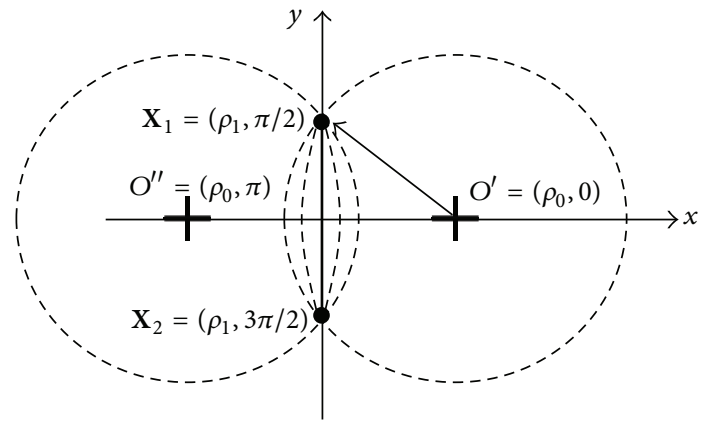

FIGURE 23: Minimum convex source region $B_{\text {min,conv }}$ of the far field of two point sources, shown here as a thick straight line uniting the two sources.

Having computed the minimum convex source region of the two point sources, we address next the respective minimum source region. First we note that the result (4) holds for any choice of the origin of coordinates. Thus if for one origin, $O$, we denote $\mathbf{r}=(\rho, \phi)$ and $\mathbf{r}^{\prime}=\left(\rho^{\prime}, \phi^{\prime}\right)$ and for another origin, $O^{\prime}, \mathbf{r}=\left(\rho_{O^{\prime}}, \phi_{O^{\prime}}\right)$ and $\mathbf{r}^{\prime}=\left(\rho_{O^{\prime}}^{\prime}, \phi_{O^{\prime}}^{\prime}\right)$, then (4) holds and also

$$
4 i G\left(\mathbf{r}, \mathbf{r}^{\prime}\right)=\sum_{m=-\infty}^{\infty} J_{m}\left(k \rho_{<, O^{\prime}}\right) H_{m}^{(1)}\left(k \rho_{>, O^{\prime}}\right) e^{-i m\left(\phi_{O^{\prime}}-\phi_{O^{\prime}}^{\prime}\right)}
$$

where of course $\rho_{<, O^{\prime}}=\min \left(\rho_{\mathrm{O}^{\prime}}, \rho_{\mathrm{O}^{\prime}}^{\prime}\right)$ and $\rho_{>, \mathrm{O}^{\prime}}=$ $\max \left(\rho_{\mathrm{O}^{\prime}}, \rho_{\mathrm{O}^{\prime}}^{\prime}\right)$. It is possible to choose the coordinate systems $O$ and $O^{\prime}$ such that $\rho_{<}=\rho^{\prime}$ while $\rho_{<, O^{\prime}}=\rho_{O^{\prime}}$ in which case the results (4) and (B.7) give

$$
\begin{aligned}
& \sum_{m=-\infty}^{\infty} J_{m}\left(k \rho^{\prime}\right) H_{m}^{(1)}(k \rho) e^{-i m\left(\phi-\phi^{\prime}\right)} \\
& \quad=\sum_{m=-\infty}^{\infty} J_{m}\left(k \rho_{O^{\prime}}\right) H_{m}^{(1)}\left(k \rho_{O^{\prime}}^{\prime}\right) e^{-i m\left(\phi_{O^{\prime}}-\phi_{O^{\prime}}^{\prime}\right)} .
\end{aligned}
$$

Using (B.1) we found that the respective minimum circular source region $B_{\min }(O)$ is defined by the minimum radius $R_{\min }=\max \left(\rho_{1}, \rho_{2}\right)$. According to (5) and (B.2), for any source that is located in the interior of this minimum circular source region and generates the given far field, the respective field everywhere outside the minimum circular source region is given by

$$
\begin{aligned}
E_{z}(\mathbf{r} & =(\rho, \phi)) \\
& =-\frac{\omega \mu}{4} \sum_{j=1}^{2} \sum_{m=-\infty}^{\infty} \alpha_{j} J_{m}\left(k \rho_{j}\right) H_{m}^{(1)}(k \rho) e^{-i m\left(\phi-\phi_{j}\right)} \quad \rho>R,
\end{aligned}
$$

and in view of (B.8) this further implies that the field at any point inside a ball centered at another reference origin $O^{\prime}$ that is fully contained in the exterior region $\mathbb{R}^{2} \backslash B_{\min }(O)$ is defined as follows. Let $\left(\rho_{O^{\prime}}, \phi_{O^{\prime}}\right)$ represent radial and azimuthal coordinates associated with a coordinate system based on the new origin $O^{\prime} \in \mathbb{R}^{2} \backslash B_{\text {min }}(O)$. Then in view (B.8) and (B.9) for any point in $\mathbb{R}^{2} \backslash B_{\min }(O)$

$$
\begin{aligned}
E_{z}(\mathbf{r} & \left.=\left(\rho_{O^{\prime}}, \phi_{O^{\prime}}\right)\right) \\
& =-\frac{\omega \mu}{4} \sum_{j=1}^{2} \sum_{m=-\infty}^{\infty} \alpha_{j} J_{m}\left(k \rho_{O^{\prime}}\right) H_{m}^{(1)}\left(k \rho_{O^{\prime}}^{(j)}\right) e^{-i m\left(\phi_{O^{\prime}}-\phi_{O^{\prime}}^{(j)}\right)},
\end{aligned}
$$

where $\left(\rho_{O^{\prime}}^{(j)}, \phi_{O^{\prime}}^{(j)}\right)$ defines the coordinates of the source points $\mathbf{X}_{j}, j=1,2$, in the new coordinate system. By applying Main Result 2, where according to (B.10)

$$
g_{m}=\sum_{j=1}^{2} \alpha_{j} H_{m}^{(1)}\left(k \rho_{O^{\prime}}^{(j)}\right) e^{i m \phi_{O^{\prime}}^{(j)}}
$$

we easily obtain by using the asymptotics (20) the result $R_{\text {min }}^{\text {int }}=\min \left(\rho_{O^{\prime}}^{(1)}, \rho_{O^{\prime}}^{(2)}\right)$. Combining the result for the convex source region of the set of 2 point sources with this nonconvex part result we conclude that the minimum source region of the 2 point sources is the set of the 2 source points. Generalizing, the minimum source region of the collection of $N$ point sources is the set of source points. This is expected since the smallest support producing the point source field (Green's function) is the location of the point source, and the fields of point sources cannot be annulled by a finite number of point sources as is well known (nonradiating sources are continuous source distributions).

\section{Conflict of Interests}

The author declares that there is no conflict of interests regarding the publication of this paper.

\section{Acknowledgment}

This work was supported by the Air Force Office of Scientific Research under grant FA9550-12-1-0285.

\section{References}

[1] S. Kusiak and J. Sylvester, "The scattering support," Communications on Pure and Applied Mathematics, vol. 56, no. 11, pp. 15251548, 2003. 
[2] A. D. Yaghjian, T. B. Hansen, and A. J. Devaney, "Minimum source region for a given far-field pattern," IEEE Transactions on Antennas and Propagation, vol. 45, no. 5, pp. 911-912, 1997.

[3] A. J. Devaney and E. Wolf, "Multipole expansions and plane wave representations of the electromagnetic field," Journal of Mathematical Physics, vol. 15, no. 2, pp. 234-244, 1974.

[4] J. Sylvester, "Notions of support for far fields," Inverse Problems, vol. 22, no. 4, article 010, pp. 1273-1288, 2006.

[5] C. Müller, Foundations of the Mathematical Theory of Electromagnetic Waves, Springer, Berlin, Germany, 1969.

[6] T. B. Hansen and A. D. Yaghjian, Plane Wave Theory of TimeDomain Fields: Near-Field Scanning Applications, IEEE Press, Piscataway, NJ, USA, 1999.

[7] S. Kusiak and J. Sylvester, "The convex scattering support in a background medium," SIAM Journal on Mathematical Analysis, vol. 36, no. 4, pp. 1142-1158, 2005.

[8] E. A. Marengo, A. J. Devaney, and R. W. Ziolkowski, "Inverse source problem and minimum-energy sources," Journal of the Optical Society of America A, vol. 17, no. 1, pp. 34-45, 2000.

[9] A. J. Devaney and G. C. Sherman, "Nonuniqueness in inverse source and scattering problems," IEEE Transactions on Antennas and Propagation, vol. 30, no. 5, pp. 1034-1037, 1982.

[10] R. Potthast, J. Sylvester, and S. Kusiak, "A "range test" for determining scatterers with unknown physical properties," Inverse Problems, vol. 19, no. 3, pp. 533-547, 2003.

[11] H. Haddar, S. Kusiak, and J. Sylvester, "The convex backscattering support," SIAM Journal on Applied Mathematics, vol. 66, no. 2, pp. 591-615, 2006.

[12] R. Potthast, "A survey on sampling and probe methods for inverse problems," Inverse Problems, vol. 22, no. 2, pp. R1-R47, 2006.

[13] G. B. Arfken and H. J. Weber, Mathematical Methods for Physicists, Elsevier Academic Press, 6th edition, 2005.

[14] C. A. Balanis, Advanced Engineering Electromagnetics, John Wiley \& Sons, New York, NY, USA, 1989.

[15] E. A. Marengo, "A new theory of the generalized optical theorem in anisotropic media," IEEE Transactions on Antennas and Propagation, vol. 61, no. 4, pp. 2164-2179, 2013. 


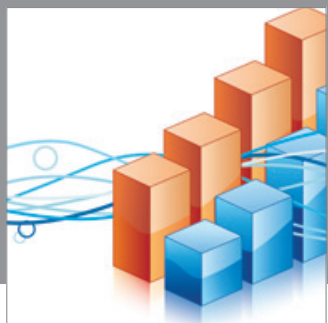

Advances in

Operations Research

mansans

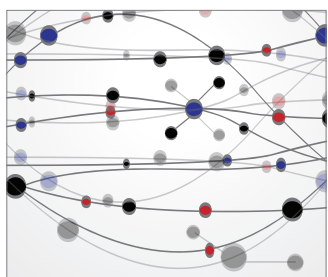

The Scientific World Journal
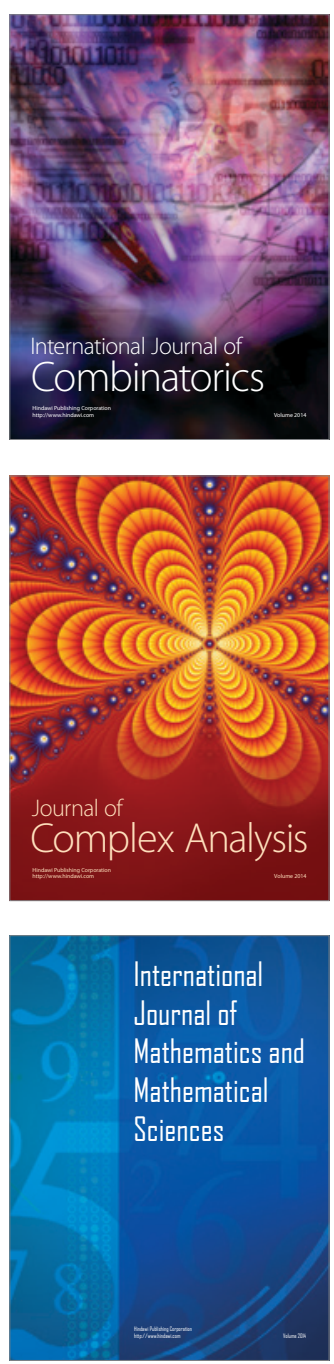
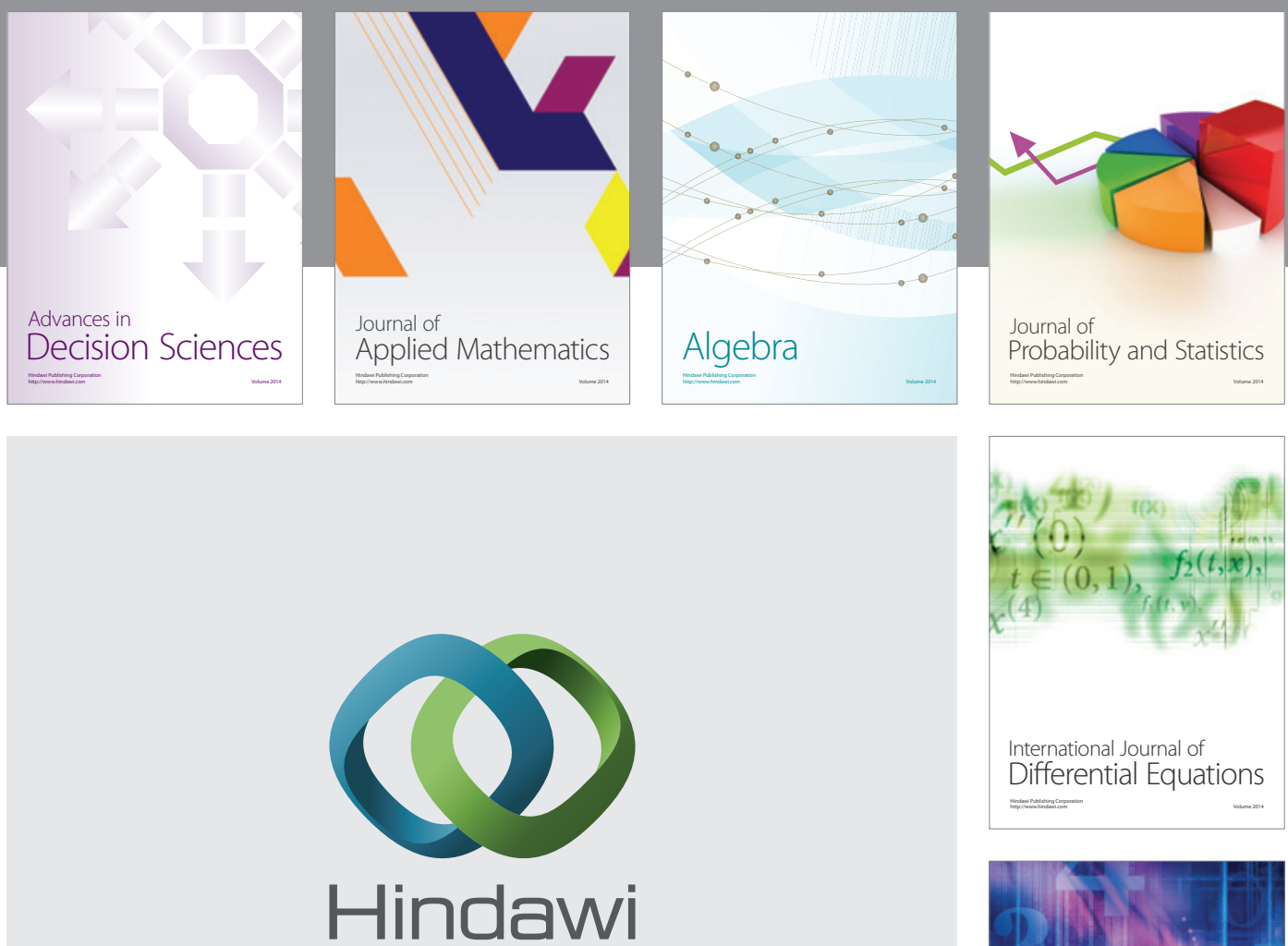

Submit your manuscripts at http://www.hindawi.com
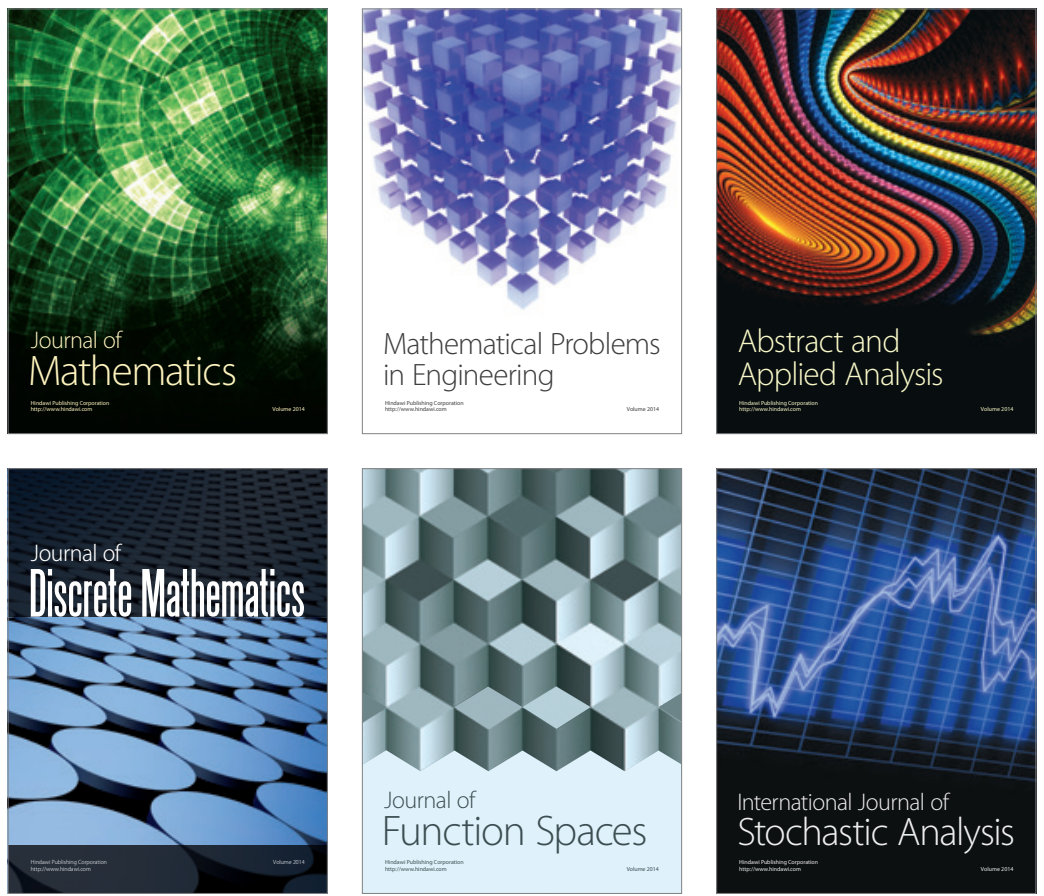

Journal of

Function Spaces

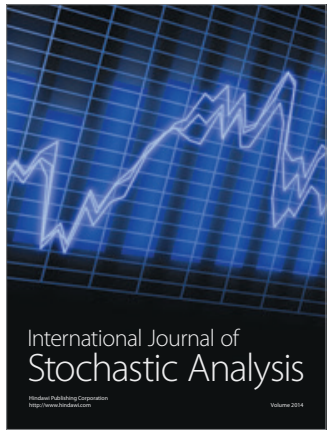

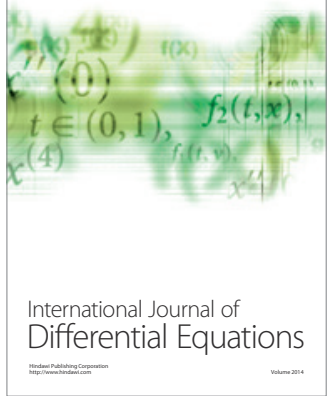
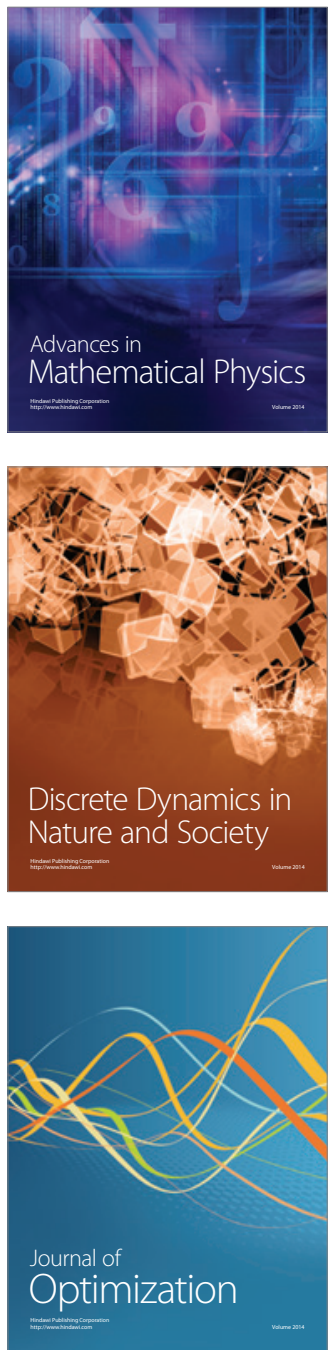